\title{
El «momento mutualista» en la formulación de un sistema de protección social en Argentina: socorro mutuo y prevención subsidiada a comienzos del siglo $\mathrm{XX}$
}

\author{
por \\ Pilar González Bernaldo de Quirós \\ Université Paris Diderot, Sorbonne Paris Cité - ICT ${ }^{1}$
}

Este trabajo está destinado a estudiar las prácticas de socorro mutuo de los extranjeros y su papel en la difusión de un pensamiento solidarista que proporcionará un conjunto de herramientas conceptuales y técnicas esenciales para las reivindicaciones de protección por parte de los trabajadores asi como para la formulación de los primeros proyectos de creación de un sistema nacional de seguro social. Ello me permite destacar la dimensión transnacional de la construcción del campo de la protección social. La primera parte del artículo está destinada a dar cuenta de esta realidad empírica a partir de un trabajo de fuentes cuantitativas y cualitativas y de archivos públicos y privados. La segunda pone en evidencia los mecanismos concretos de la circulación de saberes. El artículo analiza en particular los diferentes congresos mutualistas que se organizan en Buenos Aires durante las dos primeras décadas del siglo XX y el impacto de la visita de Léopold Mabilleau, intelectual francés, experto en mutualidades y «apóstol» del solidarismo.

Palabras Clave: Socorro mutuo; Solidarismo; Circulación de saberes; Estado social; Argentina; Siglo $X I X-X X$.

${ }^{1}$ Este artículo es parte de la investigación «Construcción del Estado social en una sociedad de inmigración», realizada en el marco del proyecto "Circulación internacional de saberes y prácticas institucionales en la consolidación del Estado Social en Argentina, 1910-1970» HAR 2009-13555, coordinado por Ricardo González Leandri y financiado por el programa I+D del Ministerio de Economía y Competitividad del gobierno de España. Versiones preliminares de esta investigación fueron presentadas en el Instituto de América Latina de la Freie Universität Berlin y en el seminario de Historia Social del Colegio de México. Agradezco a Stefan Rinke y a Clara Lida, así como a sus estudiantes, por sus comentarios. 
La historia de las políticas sociales ha conocido en estas últimas décadas un interés creciente por parte de los historiadores, suscitado por la crisis de los sistemas de protección social que habían sido establecidos durante la segunda post-guerra ${ }^{2}$. Más de dos décadas han pasado y ya disponemos hoy de una serie de trabajos que estudian, desde problemáticas y perspectiva cronológica amplias, los procesos de construcción del Estado social, abordados tanto desde el campo laboral como en los de la salud pública, la educación o el derecho ${ }^{3}$. Más recientemente, una nueva línea de investigación, en la interfase entre la historia del Estado y la historia intelectual, ha permitido abrir un abanico de nuevas problemáticas vinculadas al estudio de los mecanismos de circulación y recepción de ideas, a los procesos de institucionalización de saberes sobre la sociedad y su relación con la consolidación del Estado ${ }^{4}$. A pesar de la diversidad de perspectivas de análisis y de problemáticas privilegiadas por estas investigaciones, todas ellas están atravesadas por la problemática de la metamorfosis del estado liberal en un estado social de derecho, proceso que todos datan entre finales de siglo XIX y el gobierno de Juan D. Perón. La investigación que aquí presentamos se inscribe también en esta vasta problemática, abordada a partir del estudio del desarrollo de prácticas de previsión solidarias en Argentina. A través de este se busca dar cuenta de cómo se van introduciendo en las viejas prácticas de socorro nuevos lenguajes y representaciones de lo social que atestiguan las conexiones y circulación de doctrinas y conocimientos sociales y técnicos entre los sectores reformistas provenientes de diferentes ámbitos - principalmente universitarios y políticos-, la dirigencia del socorro mutuo - compuesta de nativos y extranjeros - y las redes reformistas internacionales. Es en el marco de estas conexiones donde se imagina y postula el socorro mutuo como base de un sistema de prevención subsidiada, que implica tanto a trabajadores como al Estado, y que permite ofrecer una respuesta funcional

2 El trabajo de Mesa-Lago tuvo también el efecto de lanzar esta problemática en América Latina. Ver Meso-Lago, 1978. Sobre las reformas estructurales de los años 90 en Argentina, ver Lo Vuolo y Barbeito, 1994; Alonso, 2000. La reflexión que desata la crisis del sistema de protección social estará marcada en Francia por los dos ensayos de Pierre Rosanvallon (ver Rosanvallon, 1981 y 1995) y la tesis de Robert Castel (ver Castel, 1999). Sobre la historia de las políticas sociales en Argentina, ver Lvovich y Suriano, 2006; Suriano (comp.), 2000.

${ }^{3}$ Optamos por hablar de Estado social y no de Estado providencia, siguiendo en este punto a Robert Castel, 1999.

${ }^{4}$ La problemática de la circulación de nuevos saberes sobre la sociedad y la metamorfosis del Estado en Argentina ya estaba en el centro de la tesis de Eduardo Zimmermann, publicada en 1995. Ver Zimmermann, 1995; 2005. Un estado de la cuestión sobre la historiografía de saberes de Estado se encuentra en Plotkin y Zimmermann (comps.), 2012: 9-28. 
a la objeción liberal al derecho de protección ${ }^{5}$. La investigación se centra en las dos primeras décadas del siglo XX, momento que corresponde al gran auge del mutualismo en Argentina, pero se inscribe en un marco cronológico más amplio que va desde la crisis de 1890 a la de 1929-30, planteando la hipótesis de que estos momentos son particularmente favorables para estudiar los fenómenos de circulación de ideas, reformulaciones del contrato social y cambios institucionales ${ }^{6}$.

\section{SOCORRO MUTUO Y SEGURO DE ENFERMEDAD}

La historia del mutualismo en Argentina aún queda por hacerse, a pesar del importante desarrollo que conoce este tipo de prevención solidaria ${ }^{7}$. Cierto es que la historia social había destacado la existencia de este tipo de forma asociativa, pero los estudios buscaron en ellos la génesis del movimiento obrero y poco se detuvieron en las funciones de socorro, sobre todo si estas funcionaban sobre una base policlasista ${ }^{8}$. En los años 80 , la historiografía

${ }^{5}$ La investigación se funda en fuentes estadísticas, archivos y libros de actas de algunas de las sociedades de socorros mutuos, documentación proveniente del Museo Social Argentino, del Boletín del Departamento Nacional del Trabajo y de la prensa periódica nacional y extranjera.

${ }^{6}$ Se trata de una de las hipótesis de trabajo del proyecto de investigación sobre «Circulation internationale des savoirs et pratiques institutionnelles dans la consolidation de l'Etat social en Argentine, 1920-1970». Ver Rosenvallon, 1981: 29. Ver también las contribuciones de Plotkin, Caravaca, Zimmerann y Daniel en este mismo dosier.

${ }^{7}$ Un desarrollo similar al que se conoce en Francia, país reputado por ser tierra del mutualismo. Una comparación entre los dos países en González Bernaldo, 2008. Sobre desarrollo de las sociedades de socorro mutuo como forma de seguro médico en Argentina ver Belmartino, Bloch, Persalli y Quiroga, 1987. Belmartino, 2005. Munck, 30/3 (1998): 573-590. Thompson, enero de 1994: 53. No existe aún una historia general del mutualismo en América Latina, lo que explica que en los esfuerzos colectivos para pensar comparativamente este desarrollo del mutualismo mundial el caso argentino esté ausente, a pesar de ser América Latina la región en que ha conocido un mayor desarrollo. Ver Linden, Dreyfus, Gibaud y Lucassen, 1996. Sobre el mutualismo en diferentes países de América Latina ver Sordo Cerdeño, 33/1 (129) (1983): 72-96. Castro, 2002: 195-221. García-Bryce, 59/3 (2003): 325-345.

${ }^{8}$ Los primeros son los especialistas del mundo obrero quienes buscaron -en una visión bastante evolutiva- los primeros indicios de organizaciones de clase y destacaron la presencia de extranjeros en las organizaciones obreras. El marco de análisis presuponía que el inmigrante se confundía con los nacionales en una misma cultura obrera y un mismo combate de clase. Ver Marotta, 1961; Oddone, 1949. Algunos, como Godio, van a señalar sin embargo las tensiones entre movimiento obrero e identidad étnica. Ver Godio, 1985. Siempre en el marco del estudio de la clase obrera, Ronald Munck señala la especificidad y autonomía del movimiento mutualista respecto al sindicalismo y la necesidad de destinarle un estudio específico en Munck, 30/3 (1998): 573-590. Gandolfo retomará esta problemática proponiendo un corto 
migratoria interesada en el asociacionismo étnico descubre el importante desarrollo de la experiencia mutualista, pero estos estudios quedan en buena medida encapsulados en el debate sobre el papel que juegan en la integración de los inmigrantes en la sociedad de acogida ${ }^{9}$. En los mismos años, una renovada historia política también repara en el desarrollo de este tipo de experiencia asociativa, pero buscando en ella indicadores de la existencia de una esfera pública y del desarrollo de prácticas democráticas ${ }^{10}$. Como es de esperar, los estudiosos sobre historia de la salud han prestado un mayor interés a la dimensión mutualista aunque este aspecto ocupa un lugar marginal en la renovación del área de la historia social y cultural de la salud, con la excepción de los trabajos de Susana Belmartino sobre historia de los sistemas de atención médica en Argentina ${ }^{11}$.

Recordemos a grandes rasgos las etapas del desarrollo del socorro mutuo en Argentina, cuya génesis nos remite al nuevo paradigma, que introducen los estados liberales, de libertad sin protección ${ }^{12}$. La primera sociedad de socorros mutuos (SSM) que conocemos fue creada en 1832, y no es casual que se trate de una pequeña «sociedad mutual de beneficencia de los artesanos franceses» ${ }^{13}$. Desde la caída de Rosas en 1852 hasta la instauración de la primera presidencia nacional en 1862, van a crearse en Buenos Aires alrededor de doce sociedades con objetivos de asistencia y ayuda mutua, de las cuales siete son claramente creadas por y para extranjeros, organizadas según sus orígenes nacionales ${ }^{14}$. Junto a ellas aparece la primera SSM por oficio, la Tipográfica, y las primeras que reúnen a la población de origen africano ${ }^{15}$. A

pero muy sugestivo trabajo sobre articulación entre fidelidades étnicas y conflicto de clases. Gandolfo, 1992: 311-332.

${ }^{9}$ El artículo de Samuel Baily sobre las asociaciones de italianos, publicado en 1982, juega sin duda un papel motor en el renovado interés de las sociedades mutualistas como espacio de preservación o invención de identidades. Ver Baily, 21/84 (1982: 485-514). Sabato, 1990: 350-366. Devoto, 1992; 1994. Devoto y Mínguez (eds.), 1992: 169-188. Fernández, III/8, 1987; 1992. Una notable excepción la constituye José Moya quien analiza el mutualismo español en un marco de análisis más flexible que el de su maestro Baily. Ver Moya, 1998. Ver también el balance de ese debate veinte años después en Devoto y Otero, 17/50 (2003): 181-228.

${ }^{10}$ Fue el caso de mi tesis doctoral, defendida en 1992. González Bernaldo de Quirós, 2001. En esta perspectiva, ver el importante aporte de Carlos Forment (Forment, 2003).

${ }^{11}$ Una síntesis del estado de la cuestión en este campo se encuentra en Armus y López Denis, 2011: 424-453; Belmartino, Bloch, Persalli y Quiroga, 1987. Otros trabajos previos ya habían reparado en el mutualismo como forma de seguro social (Passanante, 1987).

12 Ver el clásico estudio de Robert Castel (Castel, 1995).

13 Ver González Bernaldo de Quirós, 2001.

${ }^{14}$ Idem.

15 Idem. Para las sociedades africanas, ver Andrews, 1989: 180-181. 
partir de entonces, este tipo de organización conocerá un desarrollo importante, principalmente en la región litoral y en zonas urbanas.

No disponemos de fuentes cuantitativas que permitan trazar con certeza el desarrollo de este tipo de asociaciones. Muchas de ellas fueron de vida efímera o institucionalmente precaria, de modo que los datos que brindan las fuentes censales son necesariamente incompletos. A partir de fuentes estadísticas y datos obtenidos a través del cruce de fuentes cualitativas podemos trazar un esbozo de la dinámica del desarrollo de las sociedades de socorro mutuo en la ciudad de Buenos Aires desde 1852 hasta 1927.

\section{Gráfico $1{ }^{16}$. Sociedades de Ayuda Mutua creadas en Buenos Aires 1862-1927}

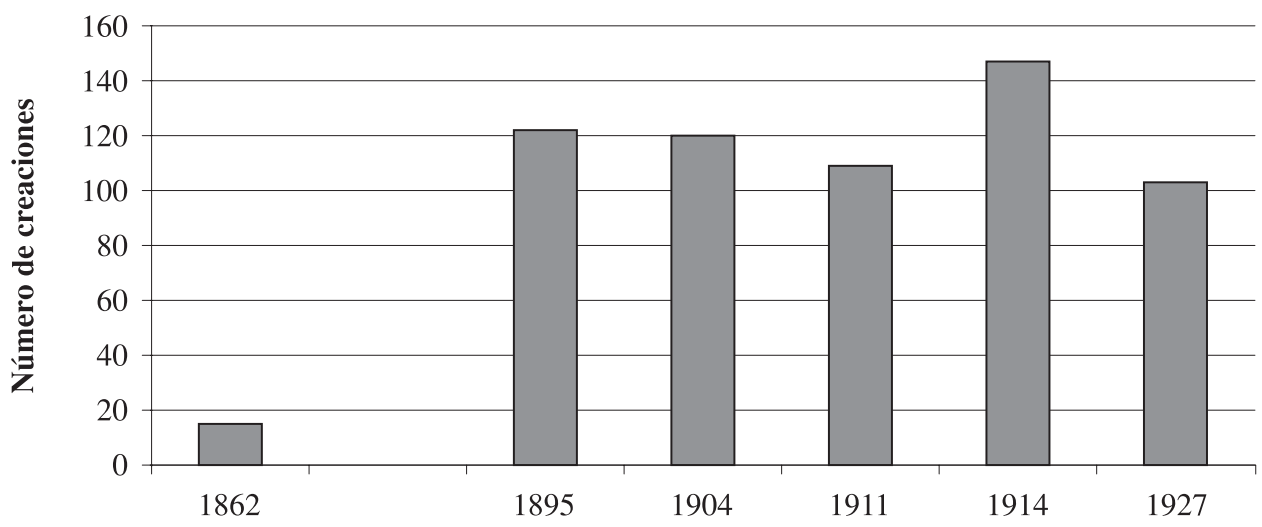

El cuadro muestra un pico de creación de sociedades para el año 1914, cuando se realiza el tercer censo nacional de la República Argentina ${ }^{17}$. Entonces se cuantifican 1202 sociedades de socorro mutuo que funcionan en todo el territorio con 507.637 miembros (dentro de estas cifras se incluyen los 70 Círculos Obreros — asociaciones de socorros mutuos católicas - con 23.920

${ }^{16}$ Las cifras deben tomarse con bastante precaución pues las fuentes utilizadas son de naturaleza diferente. Por otro lado, incluso cuando las fuentes son homogéneas -como para el caso de los censos nacionales o municipales- existen errores en la catalogación, así como también sociedades no registradas. El gráfico tiene el mérito de dar una idea de la importancia del fenómeno y tipo de evolución. Fuentes: González Bernaldo de Quirós, 2001; Censo de 1895; Censo de 1904; Encuesta del Departamento Nacional del Trabajo de 1911, Censo Nacional de 1914, Censo de Mutualidades de 1927.

17 Tercer Censo Nacional, Comisión Nacional del Censo (TCN), 1914, Buenos Aires, 1916-1919, vol.10, 91. 
socios y se excluyen las sociedades comerciales) ${ }^{18}$. Si confrontamos estas cifras con la de la población activa en la misma época nos encontramos que el mutualismo tiene entonces en Argentina una tasa de penetración similar a la francesa. Es decir, más de $16 \%$ de la población activa se beneficia de la protección que brinda el socorro mutuo. Cierto es que Augusto Bunge revisa las cifras del censo hacia la baja, excluyendo a los círculos de obreros y otras «falsas mutuales», y llega a un total de 300.000 mutualistas ${ }^{19}$. Incluso aceptando estas rectificaciones, la tasa de penetración de 9,6\% sigue siendo elevada para la época ${ }^{20}$.

Claro está que este tipo de análisis de cifras agregadas esconde realidades muy diferentes. Primero, en términos de distribución geográfica. Partiendo siempre de los datos del censo de 1914, observamos que dos de cada tres mutuales se encuentran en la ciudad y provincia de Buenos Aires. Si agregamos la zona sur de la vecina provincia de Santa Fe, los resultados son aún más espectaculares: cuatro de cada cinco SSM funcionan en esta región del litoral atlántico ${ }^{21}$. Ello se traduce en una tasa de penetración del mutualismo que alcanza el tercio de la población activa ${ }^{22}$.

Los datos del censo también dan cuenta de una desigual repartición según la nacionalidad de sus miembros, como lo muestra el gráfico 2 .

Las sociedades que el censo registra como extranjeras - cifras que excluyen las «cosmopolitas», que reúnen extranjeros y argentinos, muchos de primera generación migratoria - representan entonces más del $70 \%$ del conjunto de las SSM existentes. Así lo afirma Domingo Borea en su introducción a los datos del censo de 1914: «el mutualismo es fundamentalmente extranjero» ${ }^{23}$. Pero podemos también distinguir importantes diferencias entre las colectividades. Así, los italianos parecen más sensibles al desarrollo de este tipo de forma de mutuo socorro - las sociedades italianas representan $38,5 \%$ del total- Siguen en orden de importancia las españolas, con 250

${ }^{18}$ Los datos del censo no son necesariamente completos ya que existen una serie de pequeñas sociedades que integran la función de socorro mutuo que no figuran en él.

19 Intervención al Congreso de Mutualidades de 1918. Ver Museo Social Argentino, Congreso de Mutualidad, Buenos Aires, 1918: 80.

${ }^{20}$ Según evaluaciones de A. Gueslin, la tasa de penetración de la mutualidad adulta pasa de 2,7\% de activos en 1856 a 16,4\% en 1911. Gueslin, 1987: 246. Las cifras sobre población económicamente activa las tomamos de Germani, 1987: 118. El censo de las mutuales se encuentra en Borea, TCN: 91.

${ }^{21}$ Idem.

22 Cálculos realizados a partir de las cifras del censo de 1914 y los cálculos de Guy Bourdé sobre población activa de la ciudad de Buenos Aires. Ver Bourdé, 1974.

${ }^{23}$ Borea, TCN. 
Gráfico 2. SociedAdes DE AYUdA MUTUA DE LA CAPITAL SEGÚN NACIONALIDAD DE LOS SOCIOS (CENSO 1914)

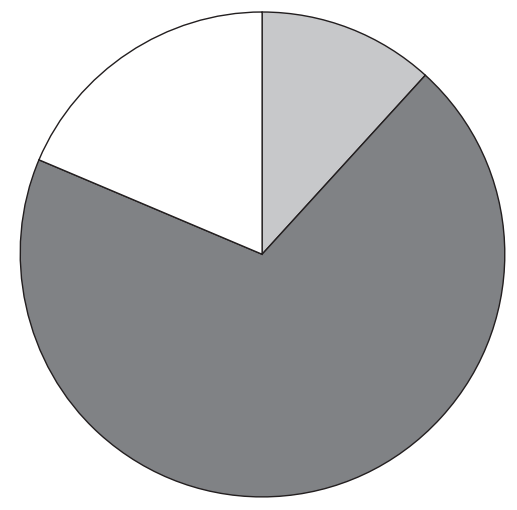

sociedades que representan $20,7 \%$ del total ${ }^{24}$. Muy por detrás se encuentran las catalogadas como «argentinas», que representan solo un $14 \%$ del conjunto. Incluso si confrontamos estas cifras con la población de cada uno de los grupos citados, se desprende que la tasa de penetración de la mutualidad es sensiblemente más alta entre extranjeros que entre nacionales ${ }^{25}$.

Gráfico $3^{26}$. Sociedades de SOCORRO MUtUO DE CAPITAL, SEGÚN NACIONALIDAD DE LOS MIEMBROS 1914

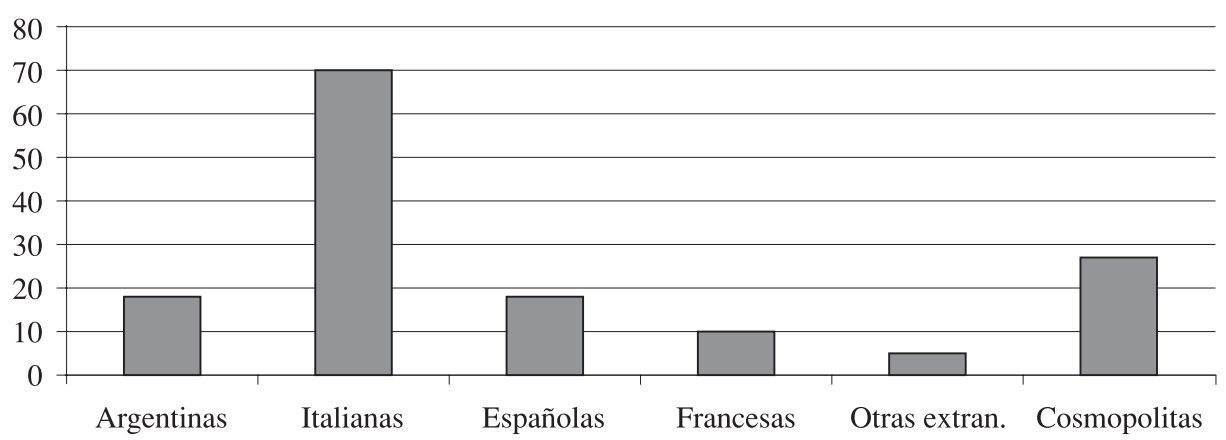

Las diferencias considerables entre el desarrollo del mutualismo italiano y el español no traducen una distancia similar en número de adhesiones, sino

\footnotetext{
${ }^{24}$ Idem.

${ }^{25}$ Idem. Germani, 1987.

${ }^{26}$ Ver el TCN.
} 
que dan cuenta de una tendencia a una mayor dispersión del mutualismo italiano respecto al español. Los especialistas de las migraciones vinculan este fenómeno a la fuerte conflictividad política-ideológica que caracteriza a la colectividad italiana ${ }^{27}$.

Gráfico 4. Total de SOCIOS DE SOCIEDADES DE AYUdA MUTUA DE LA CAPITAL (CENSO DE 1914)

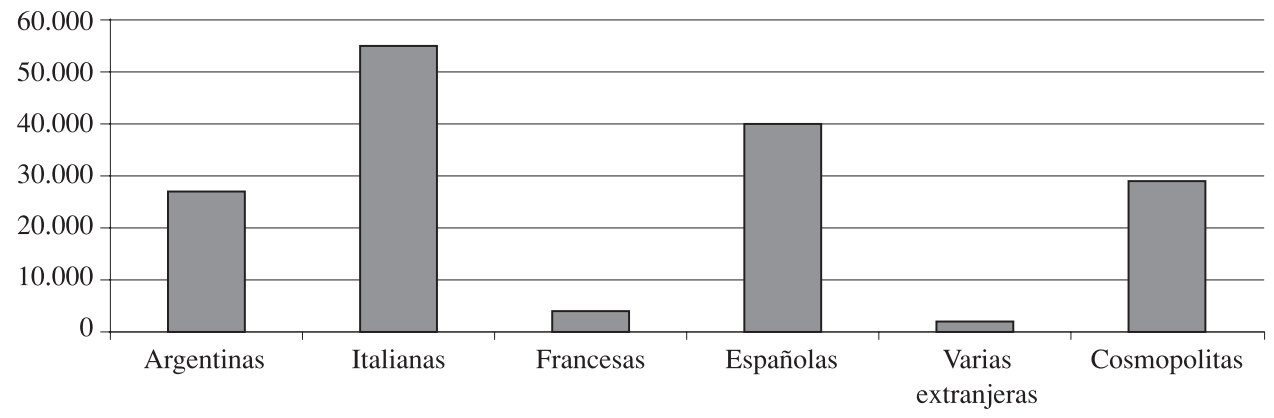

Sin embargo, si calculamos el número de adherentes de cada nacionalidad respecto a la población total de la colectividad, notamos que los italianos son los que comparativamente parecen más atraídos por las sociedades de socorro mutuo étnicas: el $17,20 \%$ de los italianos se adhieren a una sociedad de socorro mutuo de la colectividad, mientras que estas cifras descienden a $13,24 \%$ para los españoles y $13,71 \%$ para los franceses ${ }^{28}$. Pero ello no traduce necesariamente una diferencia en la protección, ya que muchos de ellos participan en asociaciones «cosmopolitas» o confesionales. Es difícil cuantificar la proporción de los inmigrantes que optan por adherirse a una sociedad de tipo cosmopolita, pero no es insensato considerar que alrededor de la misma cantidad de miembros que se adhieren a una sociedad étnica lo hacen a una que reúne a sus miembros según criterios distintos a los del origen nacional ${ }^{29}$.

Debemos igualmente destacar diferencias importantes dentro de las sociedades de una misma colectividad. Si tomamos el caso de sociedades españolas, ¿cómo tratar con criterio de igualdad la Asociación Española de Socorros

${ }^{27}$ Sobre conflictividad en la colectividad italiana ver Devoto, 1991: 144-166; 2008: 165232. Cibotti, serie 3/2 (1990).

${ }^{28}$ TCN; Bourdé, 1974.

29 Ello explicaría la diferencia entre los cálculos de tasas de penetración del mutualismo entre la población activa total $(30 \%)$ y los porcentajes de participación a las sociedades de la colectividad (entre 13\% y 17\%). 
Mutuos (AESM), que concentra en 1914 más de la mitad del total de adherentes de esta nacionalidad (22.204 sobre un total de 40.054), y la Asociación Canaria, con 240 socios? Los gráficos $n^{\circ} 5$ y n $^{\circ} 6$ dan cuenta de esta diversidad y de las diferencias en las características que presenta el socorro mutuo en ambas colectividades.

Gráfico $5^{30}$. Número de SOCIOS DE las SOCIEDADES ITALIANAS DE SOCORRO MUTUO EN 1914

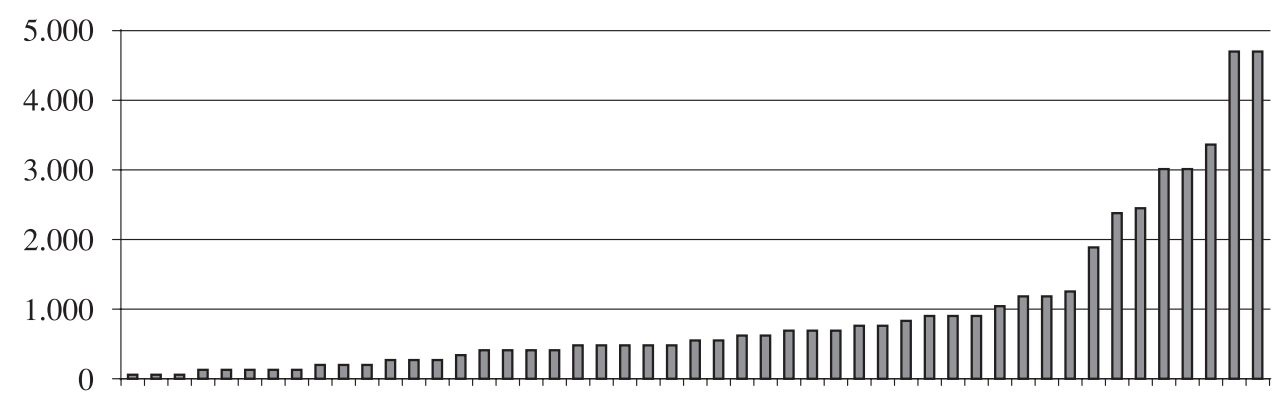

GRÁFICO $6^{31}$. NÚMERO DE SOCIOS DE LAS SOCIEDADES DE AYUDA MUTUA ESPAÑOLAS

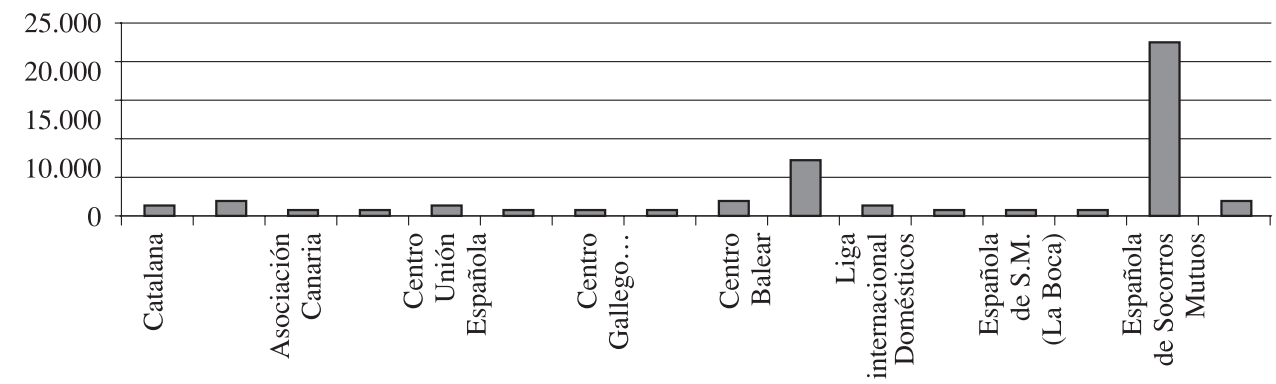

Este tipo de contrastes ha sido ya destacado por los especialistas de estudios migratorios, pero han sido analizados como una manifestación de lógicas de identidad étnica según la clásica tesis de Samuel Baily ${ }^{32}$. Ahora bien, si el consumo de bienes culturales compartidos en la tierra de inmigración no es ajeno a la atracción que puede ejercer este tipo de socieda-

\footnotetext{
${ }^{30}$ Ver TCN.

31 Idem.

${ }^{32}$ Baily, 21/84 (1982): 485-514.
} 
des, la introducción de la variable «sistema de seguro médico» permite una interpretación diferente de estos datos. Para ello es necesario primero recordar que las cuotas que se exigían para ser considerado miembro de una sociedad - tanto étnica como cosmopolita organizada en torno a un mismo oficio - iban destinadas mayoritariamente a ofrecer un sistema de socorro. $\mathrm{Si}$ en un comienzo las funciones estaban bastante diversificadas — salud, educación, subsidios en caso de pérdida de empleo, servicio de sepelios, ayuda a familiares en caso de defunción, etc. - y estaban más próximas de la beneficencia que del seguro - las primeras asociaciones se denominan de «beneficencia y socorro»-, hacia fines del siglo XIX estas tienden a especializarse en el seguro médico, ofreciendo tanto atención médica como medicinas, ramos que poco a poco ocupan el primer lugar de los gastos de estas sociedades.

Susana Belmartino muestra, a partir de un análisis de la encuesta sobre sociedades de ayuda mutua llevada a cabo por el Departamento Nacional del Trabajo en 1911, que más de un $80 \%$ de las sociedades cubren la atención médica, y el $86 \%$ de sus socios recurren a este tipo de prestaciones ${ }^{33}$. Siguen en orden de importancia los medicamentos, cuyos gastos son asegurados por el $80 \%$ de las sociedades, recurriendo a esta cobertura más de $70 \%$ de los adherentes. Vienen luego los subsidios, para los cuales disponemos los datos del censo de 1914, cubiertos por más del $68 \%$ de las sociedades y a los que acceden más del $65 \%$ de los socios. Si consideramos que la distribución del gasto está directamente vinculada a la demanda de los adherentes, debemos concluir que la principal función de las SSM era la de ofrecer un seguro médico. Este aspecto es anunciado tempranamente por el socialista español Victory y Suárez en las páginas del Imparcial Español: «Hoy el deseo de pertenecer a una de estas sociedades no exige más que un cálculo frío: pagaré 60 pesos de cuota de ingreso, 20 pesos de cuota mensual, y como beneficio recibiré 20 pesos diarios en caso de enfermarme, tendré atención médica y remedios gratuitos...» ${ }^{34}$. Pero este frío cálculo no parece ser una especificidad de los inmigrantes. Semejante ecuación parece también guiar a los trabajadores que se adhieren a los círculos obreros, y aquellos que buscaban objetivos morales más que materiales reconocen con pesadumbre «que ha llegado a desaparecer de la mente de ciertos asociados la creencia de que ellos puedan responder a fines superiores o extraños a los simples del socorro y asistencia en caso de

${ }^{33}$ La encuesta es bastante exhaustiva ya que reúne datos de 108 SSM, cuando el censo de 1904 registra 117 en Capital y el de 1914, 144. Ver Belmartino, Bloch, Persalli y Quiroga, 1987:17-18.

${ }^{34}$ Ver El Imparcial, 5 de mayo de 1864, citado por Moya, 2004: 309. 
enfermedad ${ }^{35}$. No dice otra cosa uno de los miembros de la Asociación Obrera de Socorros Mutuos cuando declara: «Para atenuar la miseria que acarrean las enfermedades tenemos las asociaciones de socorros mutuos; para defendernos de las invasiones del capital, las sociedades gremiales de resistencia; para prevenir los paros forzosos del trabajo, las Cajas de Parados.... ${ }^{36}$.

Aunque estos datos necesitan ser matizados, entre otras cosas debido a la presencia de «socios honorarios» que hacen pensar que no todos los que pagan una cuota lo hacen persiguiendo los mismos objetivos, ellos muestran que las sociedades de socorro mutuo, tanto étnicas como confesionales y obreras, cumplen como principal función la de ofrecer una cobertura médica. A cambio de la cotización mensual — que representa más o menos el pago de un jornal de un obrero no calificado-, estos buscan tener acceso a la atención médica, al consumo de fármacos, y secundariamente a disponer de un pequeño subsidio en caso de interrupción de actividad laboral. Esta protección parece ser particularmente vital para los inmigrantes, puesto que en su caso la enfermedad no implica únicamente la pérdida temporal del salario. Sin densas redes familiares en el país de acogida, la enfermedad pone en peligro el proyecto migratorio que puede implicar a la familia que ha quedado en el país de origen. De modo que no es llamativo constatar que este tipo de prácticas de prevención son particularmente desarrolladas entre los inmigrantes con un cierto nivel de ingresos ${ }^{37}$. Así como ya lo señala José Moya, «el estímulo para la actividad asociativa reside menos en el entorno cultural del emigrante o de los hábitos cívicos de sus anfitriones que en el propio proceso migratorio» ${ }^{38}$.

Cierto es que la gran diversidad de situaciones que hemos evocado también se traduce en importantes diferencias en cuanto a la solidez financiera de cada una de estas sociedades y, por consiguiente, en los diferentes niveles y calidades de las prestaciones en atención médica, medicinas y subsidios. Si es difícil evaluar a partir de los datos disponibles el tipo de protección médica efectiva de que se beneficiaron los adherentes, las distintas estrategias por las que optan las sociedades muestran que por un lado la prestación de servicios fue una exigencia cada vez más reiterada de los adherentes, y por el otro que el incremento del gasto de atención médica y de medicinas colocaba a las sociedades en situación de permanente desequilibrio financiero. Pero

\footnotetext{
35 Ver Niklison, 46 (Buenos Aires, 1920): 199-200.

${ }^{36}$ Ver la revista La Mutualidad (1904), citado por Belmartino, Bloch, Persalli y Quiroga, 1987: 13.

37 Ver Primer Congreso de la Confederación española en Argentina. Buenos Aires, 1913.

38 Ver Moya, 31/5 (2005): 833-864.
} 
incluso aceptando la idea de diferencias en las prestaciones, debemos destacar el hecho irrefutable de que las sociedades de socorro mutuo hacen posible - dentro de una gran diversidad de situaciones - un consumo de medicina clínica y de medicamentos antes solo accesible a un sector muy reducido de la sociedad que podía hacer frente a este tipo de gastos. Sin disponer por el momento de elementos suficientes para examinar la incidencia directa sobre el estado de salud de la población, podemos postular que este consumo incide sobre la cultura somática de los asociados, que provoca una mayor percepción de las sensaciones mórbidas y que los induce por consiguiente a una mayor atención y cuidado del cuerpo, que se traduce en el desarrollo de prácticas destinadas a la prevención y al acceso a la medicina clínica ${ }^{39}$. En otros términos, el socorro mutuo alimenta la demanda de salud a la que responde en buena medida el propio mutualismo. Este mecanismo de realimentación puede explicar tanto la vitalidad del movimiento de socorro mutuo como su fragilidad, ya que la especialización de la demanda se acompaña de un importante aumento de los costos de la medicina, particularmente de los medicamentos, que aumentan en proporciones más importantes que los asociados, colocando a las sociedades en situación de gran fragilidad financiera $^{40}$. Si a ello agregamos los diferentes cuadros epidémicos y la crisis económica, que se traduce en aumento del desempleo y que repercute sobre la recaudación de las cotizaciones, podemos adivinar las dificultades que encontraron las SSM para responder adecuadamente a una demanda que ellas mismas estaban alimentando.

Este proceso ha sido bien documentado para el caso de la Sociedad Obrera de Socorros Mutuos ${ }^{41}$. Pero podemos también constatarlo, con ciertas variantes, en pequeñas sociedades étnicas. Vemos el caso de la catalana Montepío de Montserrat ${ }^{42}$. Se trata de una sociedad creada en Buenos Aires en 1857 por un puñado de catalanes, que cuenta con alrededor de 900 adherentes en 1890 y que alcanza es su momento más fausto a fines de los años 20 del siglo XX los 1500 miembros $^{43}$. Durante la crisis económica de 1890 la sociedad debe hacer frente a una situación doblemente crítica, provocada por el aumento de morosos, unida a la pérdida de sus fondos depositados en el Banco de la

\footnotetext{
39 Sobre cultura somática ver Boltanski, 26/1 (1971): 205-233. Didier Fassin, 1996: 110111.

${ }^{40}$ Ver sobre este punto Belmartino, 2005.

${ }^{41}$ Idem.

${ }^{42}$ Un estudio detallado de esta sociedad ha sido presentado en González Bernaldo de Quirós, 2008.

${ }^{43}$ Cuantificación hecha a partir de los datos encontrados en el Archivo de la sociedad Montepío de Montserrat. Ver González Bernaldo de Quirós, 2008.
} 
Provincia, que acababa de declararse en bancarrota ${ }^{44}$. Si los catalanes logran sobrellevar una situación de semi quiebra ello se debe, según consignan las memorias de la comisión directiva, a que un gran número de enfermos ha renunciado a los subsidios ${ }^{45}$. Las actas dan cuenta de cómo en diferentes momentos de desequilibrio financiero, la sociedad sobrelleva la crisis a través de este mecanismo, que responde más a una lógica de beneficencia intracomunitaria, vinculada a la construcción de liderazgo étnico, que a la de seguro médico ${ }^{46}$. Pero para principios de siglo constatamos un cambio significativo: la crisis proviene ahora tanto de la situación económica del país como de la tendencia que tienen cada vez más los miembros a hacer uso de los servicios que la sociedad debe procurarles según los estatutos. Si en 189840 de los 78 enfermos renuncian a hacer uso de los beneficios que la cotización les permite reclamar, en 1904 estos representan solo seis de 60 enfermos $^{47}$. Si la situación de la sociedad mejora sensiblemente hacia el centenario, en parte gracias a nuevos ingresos de socios y a la operación financiera exitosa que realiza la sociedad vendiendo el Panteón de la Recoleta y comprando bonos municipales de pavimentación, la protección médica a crónicos a través de la construcción de un sanatorio especializado provoca nuevamente grandes desequilibrios ${ }^{48}$.

\section{EL SOCORRO MUTUO Y LA DOCTRINA SOLIDARISTA: HACIA UN SISTEMA DE PREVENCIÓN SUBSIDIADA}

A medida que aumentaba la demanda del servicio médico de los asociados y, junto a ella, el costo del mismo, las sociedades buscarán nuevas estrategias para hacer frente a la situación. Una de ellas consistió en firmar acuerdos entre sociedades a fin a abaratar los costos de los servicios propuestos. La idea de unión estuvo en un primer momento fundada en un diagnóstico

${ }^{44}$ Ver Societat catalana de socors mutuos, Memoria que la comissió directiva del MontePio de Montserrat presenta a sos consocios, (SCSM), Buenos Aires, 1892.

${ }^{45}$ En muchas de estas sociedades -y no solamente en las étnicas- algunos cotizantes lo eran a título de «protectores» $\mathrm{u}$ «honorarios», dando al sistema de socorro mutuo una dimensión tutelar que fue denunciada por los socialistas. En las sociedades de socorro de las colectividades extranjeras ello funcionaba como un mecanismo de consolidación de los liderazgos comunitarios. Ver SCSM, 1892.

46 Así ocurre en 1898, cuando más de la mitad de los enfermos renuncian a cobrar subsidios. En 1902 el desempleo es evocado nuevamente como responsable de la difícil situación financiera que provoca una pérdida de cotizaciones. Ver SCSM, 1898 y 1903.

${ }^{47}$ Ver SCSM, 1905.

${ }^{48}$ Ver SCSM, 1915. 
rudimentario, pero no desacertado, del problema, e implicaba una solución pragmática de escasa tecnicidad que consistía en amortiguar el riesgo introduciendo una economía de escala. Dado que el monto de las cuotas era el mismo para todos los adherentes adultos del mismo sexo, independientemente de la edad y los achaques, y que ningún cálculo de probabilidades parecía preceder a la fijación del monto de las subvenciones, las pequeñas sociedades eran particularmente vulnerables a los vientos del imprevisible infortunio que podía afectar a sus asociados, que venían justamente a buscar en las SSM una protección frente a este tipo de riesgos. Como hemos podido observar en los gráficos del primer apartado, muy pocas de las SSM superaban los 1000 miembros, cuando se consideraba que este era el tamaño mínimo que una sociedad debía tener para poder garantizar las funciones de protección que estipulaban sus estatutos ${ }^{49}$.

Las iniciativas destinadas a concretar uniones entre pequeñas sociedades parecen haber sido más tempranas entre las sociedades de socorro mutuo españolas que entre las italianas. Ello posiblemente pueda explicarse por el hecho de que estas últimas conocieron un menor grado de conflictividad política. Pero también se explica por las características del desarrollo del socorro mutuo en la colectividad, como se desprende del gráfico n. ${ }^{\circ} 6$. En efecto, la fuerte concentración de miembros en una de las sociedades, la Asociación Española de Socorros Mutuos (AESM) acrecienta, sin lugar a dudas, el poder de «negociación» de la AESM con otras sociedades españolas, que disponían de escaso margen de acción ${ }^{50}$, situación hegemónica que esta comenzó a perder con la creación de una mutual gallega en $1907^{51}$. En todo caso, hacia principios de siglo XX volvemos a constatar nuevas iniciativas de hermandad, cooperación, unión o fusión como respuesta a la crisis ${ }^{52}$. La novedad provine ahora de que la crisis económica, que se acompaña de una fuerte conflictividad social, coincide con una intensa circulación de nuevos saberes, solicitados por los sectores dirigentes en busca de nuevos conocimientos que brindasen

49 Los cálculos son de Argentino Acerboni, uno de los primeros actuarios de la Argentina. Ver Congreso de la mutualidad organizado por el Museo Social Argentino y celebrado en la ciudad de Buenos Aires durante los días 24-25 y 26 de marzo de 1918, 1918.

${ }^{50}$ En las memorias de la Sociedad Montepío de Montserrat podemos descubrir que esta se lamenta de no haber sido invitada a enviar delegados a la Junta Central de la Confederación de Socorros Mutuos. SCSM, 1890. Belmartino hace referencia a varias iniciativas de este tipo en Belmartino, 2005: 14 y 40. Sobre AESM ver Fernández, III/8 (1987).

${ }^{51}$ En momento de su creación, la gallega concentra $17 \%$ de todos los españoles mutualistas; en 1930 el centro gallego tenía casi mismo número de adherentes que la AESM. Ver Moya, 2004: 304-305.

${ }_{52}$ Ver Belmartino, Bloch, Persalli y Quiroga, 1987. 
respuestas «adecuadas» a la «cuestión obrera» ${ }^{53}$. Y entre estos, la doctrina «solidarista» ofrecía una filosofía que se postulaba como la superación de las dos doctrinas enfrentadas - la individualista y la colectivista-, y un método para llegar a ella: el mutualismo.

Si el término «solidaridad» comienza a difundirse en los años ochenta en Francia, en particular a través de filósofos como Alfred Fouillée ${ }^{54}$, el término adquiere reputación internacional con la tesis de Durkheim La división del trabajo social de 1893, que lo postula como ley constitutiva de la sociedad. Desde entonces, la «solidaridad» pasa a ocupar un lugar de honor en el vocabulario republicano ${ }^{55}$. Léon Bourgeois la consagra con su libro-programa «La solidaridad» publicado en francés en $1896^{56}$. Sin cuestionar el postulado liberal, este afirma que la libertad debe ejercerse en el marco de la «ley de solidaridad natural». Fundada en la evidencia sociológica de la interdependencia social - que Durkheim define como solidaridad mecánica- de ella se desprende el principio de «deuda social» que tenemos todos con las generaciones pasadas, presentes y futuras. La doctrina permite introducir, a través de la noción de deuda social, el principio de reparación hacia aquellos que en razón de esta interdependencia están más expuestos a los riesgos que implica la vida en sociedad que beneficia a todos. La ley de accidentes de trabajo es la que mejor plasma estas nuevas teorías en el ámbito del derecho. Bourgeois hace suyo el concepto de «propiedad social» desarrollado por Fouillé que permitía concebir el principio de redistribución, el cual justificaba la noción de «deuda social» sin cuestionar la propiedad individual ni fundarla únicamente en el deber moral. Los accidentes ya no son concebidos como desgracias individuales sino como riesgos sociales, que comportan para los solidaristas una amplia paleta que va desde el desempleo hasta el de la enfermedad ${ }^{57}$. Ahora bien, esta doctrina, que se erige tanto contra el individualismo como contra el Estado providencia (el concepto ya es utilizado entonces), coloca al mutualismo en el centro del

\footnotetext{
${ }^{53}$ Ver Suriano, 2010: 153-206.

${ }^{54}$ Ver Fouillée, 1884: 294. Para la difusión de la obra de Fouillée en América Latina, ver Hale, 1991: 1-64.

55 Ver Donzelot, 1984: 73-120.

56 Léon Bourgeois (1851-1925) fue un importante hombre político francés identificado con lo que Topolov denominó la «nebulosa reformista». Presidente del Consejo en 1895, varias veces ministro, del Interior, de la Instrucción Pública, del Trabajo, de la Justicia, y del Trabajo y Prevención Social. Ocupa también importantes cargos internacionales en el Tribunal de la Haya y en la Sociedad de Naciones, carrera coronada con el Premio Nobel de la Paz en 1920. Ver Bourgeois, 1896.

${ }^{57}$ Los médicos higienistas serán particularmente receptivos a esta doctrina. Belmartino, 2005 .
} 
dispositivo de promoción de la solidaridad social ${ }^{58}$. Ello explica el renovado interés que van a manifestar los sectores reformistas locales, entre los cuales encontramos universitarios, profesiones liberales, políticos y técnicos, por las tradicionales prácticas de mutuo socorro como camino posible hacia la reforma social ${ }^{59}$. Es entonces que constatamos la presencia en el debate local de referencias a autores y doctrinas que integran lo que Serge Audier califica de «nebulosa solidarista» ${ }^{60}$. Esta comprende tanto el solidarismo católico, que difunde el catolicismo social, como el cooperativismo protestante, que llega a través del interés que suscitan los trabajos de Charles Gide ${ }^{61}$, el solidarismo jurídico que encarna Léon Duguit, uno de los precursores del derecho social y que será invitado por la Universidad de Buenos Aires para dar una serie de conferencias en $1911^{62}$, y un solidarismo científico y laico sobre el cual se funda la doctrina de la solidaridad de Léon Bourgeois, conocida gracias a la acogida que se hará a sus trabajos tanto entre los juristas como también entre los médicos higienistas ${ }^{63}$.

${ }^{58}$ Esta doctrina era conocida en Argentina pero aparentemente estaba asociada al «colectivismo» si juzgamos por la reacción que tendrá el cuerpo docente frente a la primera tesis doctoral presentada por Alfredo Palacios sobre «La Miseria» en 1901, tesis rechazada por el jurado de la Facultad de Derecho y ciencias sociales. En ella Palacios sostiene que hasta que se socialicen los medios de producción, la solución a los males causantes del hambre en la sociedad argentina se encuentra en el mutualismo. «La ley de la lucha por la vida quedará tan atenuada que casi podrá decirse que no existe. En cambio, la ley de la solidaridad triunfante, establecerá el perfecto acuerdo y la benevolencia entre los hombres». Palacios, 1900 (reedición del Colegio Público de Abogados, 2004).

59 Sobre los sectores reformistas ver Zimmermann, 1995.

${ }^{60}$ Ver Audier, 2010.

${ }^{61}$ Ver Zimmernann, 1995: 83-91. Carnavaca y Plotkin, 47/187 (2007): 401-428. Plotkin y Caravaca, 21/2 (2009): 87-108, y el trabajo en este mismo monográfico.

${ }^{62} \mathrm{Su}$ segunda conferencia será destinada al tema de «La solidaridad social y la libertad individual», en la que defiende que la libertad no es un derecho sino la consecuencia de una obligación. Ver «Les conférences du Professeur Léon Duguit» en Le Courrier de la Plata. Ver en este mismo número el artículo de Eduardo Zimmermann "“Un espíritu nuevo": la cuestión social y el Derecho en la Argentina, 1890-1930».

${ }_{63}$ Bourgeois, 1998. Los médicos también han integrado este lenguaje. El médico higienista y francomasón utiliza la noción de «deuda social», central en el pensamiento solidarista, en un artículo publicado en la Semana Médica de 1905; citado por Belmartino, 2005: 100. La Liga Argentina contra la Tuberculosis va a promover la constitución de una federación de sociedades de socorro mutuo de la capital en 1902. Ver Armus, 2007. Para un período posterior ver también Recalde, 2000: 55-86. La doctrina de Bourgeois conocerán una amplia difusión gracias al viaje de su colega Léopold Mabilleau. Ello no significa sin embargo que el mutualismo sea un movimiento de inspiración únicamente francesa. Como ya lo señaló Munch, la vinculación del mutualismo con los diferentes corrientes solidaristas y más globalmente con el positivismo aún queda por estudiar. Ver Munck, 30/3 (1998). 
Dentro de las diferentes iniciativas adoptadas por los grupos reformistas, una de las más exitosas — si evaluamos el éxito en términos de visibilidad pública e impacto político - fue la invitación que hará el recientemente creado Museo Social Argentino, bajo la explícita inspiración de su homólogo francés, a un reconocido experto europeo en mutualidad, Léopold Mabilleau, entonces director de la Federación Internacional de Mutualidades y del Museo Social Francés ${ }^{64}$. Mabilleau había jugado, junto con Léon Bourgeois, un papel importante en la promulgación de la ley de mutualidades francesa de 1898, que constituía un modelo de regulación de prácticas de socorro destinado a poner término a la «imprevisión de las sociedades de previsión» — según resume un republicano francés la situación de las mutuales antes de la ley-, a través de la exigencia de un mayor rigor en la gestión financiera de las mismas ${ }^{65}$. No es entonces casual que Léopold Mabilleau haya sido invitado por el Museo Social Argentino para dar una serie de conferencias sobre mutualidad y seguro social durante el invierno austral de $1912^{66}$. Durante su estadía, Mabilleau tomará contacto con diferentes actores locales de la economía social, pero también se vinculará con las elites gobernantes, siendo incluso recibido por el presidente de la República, Roque Sáenz Peña ${ }^{67}$. Las conferencias fueron un éxito, si damos fe a los comentarios que figuran en la prensa periódica. Pero la dispar cobertura que otorgarán los periódicos a su presencia, permite sondear el desigual interés que suscita la doctrina solidarista entre los diferentes sectores implicados en el socorro mutuo. El periódico socialista La

${ }^{64}$ Léopold Mabilleau era un prestigioso universitario francés, formado en la Escuela Normal Superior de París como especialista de estudios clásicos. Su compromiso con el movimiento reformista hizo de él un gran orador de las virtudes del mutualismo. En 1897 será nombrado director del Museo Social francés, dirigiendo la institución hacia la cruzada del mutualismo. Entre 1902 y 1904 dirige la Federación Nacional de la Mutualidad Francesa logrando en el Congreso de Mutualidades de Nantes de 1904 que se llegue al compromiso de conciliar la obligación de la previsión con la práctica de la previsión libre. Pero en 1910 ya es un convencido partidario de la obligatoriedad de la previsión. Una historia del Museo Social Argentino se encuentra en Pelosi, 2000. Para su homólogo francés ver Horne, 2004: 384.

${ }^{65}$ Ver Dreyfus, Gibaud, y Gueslin, 1999.

${ }^{66}$ Durante el mes de agosto Mabilleau pronuncia seis conferencias en la ciudad de Buenos Aires sobre los temas siguientes: «Mutualismo y previsión social», «Mutualismo y socialismo», «La carestía de la vida», «La acción social de la mujer», «Unidad Nacional de un Museo Social» y «La organización de la mutualidad». Mabilleau regresa en 1913 invitado esta vez por Joaquín V. González para dar una serie de conferencias en la Universidad de La Plata. Ver Le Courrier de la Plata (agosto-septiembre, 1912); «Las conferencias del Sr Mabilleau», Boletín Mensual del Museo Social Argentino, 1, Buenos Aires, 1912.

67 «Llega Leopoldo Mabilleau», en La Prensa (5 de agosto, 1912): 7, col. 3-5; «Leopoldo Mabilleau y el Presidente Sáenz Peña. Una entrevista cordial», en El Diario (15 de agosto, 1912): 1, col. 2-3. Sobre el Museo Social en Francia ver Horne, 2004. 
Vanguardia apenas informa de su presencia en la capital y solo le destina un artículo cuando Mabilleau visita el Hogar Obrero, invitado por su presidente, el socialista Nicolás Repetto. Con cierta socarronería el periódico señala «que viene el señor Mabilleau a confirmar y, tal vez, a vigorizar la obra de los que aquí, en Argentina, trabajan desde hace quince años en pro de la cooperación libre....», subrayando el vano esfuerzo de traer a un hombre de tan lejos «para hablarnos de cosas tan sencillas...» sin dejar de revelar a sus lectores que el interés que este despierta en ciertas esferas dirigentes no es ajeno a la voluntad de presentar a la mutualidad como antídoto del socialismo ${ }^{68}$. El periódico católico El Pueblo prácticamente no da cuenta de su paso por Buenos Aires y solo lo hace para informar de la visita que este realiza al Asilo Santa Felicitas que depende de la Sociedad San Vicente de Paul ${ }^{69}$. La Prensa, El Diario y La Nación no solo cubren la noticia de manera diferente, reproduciendo en el caso del último el contenido de las conferencias. Su condición de experto de mutualidades no deja indiferentes a las colectividades extranjeras, y en particular al principal periódico de la colectividad francesa, Le Courrier de la Plata. Más allá del compromiso que el periódico dice tener con la causa mutualista, no es difícil imaginar que busca también sacar beneficio del prestigio que goza el ilustre compatriota entre las elites locales ${ }^{70}$. El gran banquete que se organiza en el Colón para darle la despedida y que reúne, según los periódicos, entre 900 y 1000 comensales, es revelador de los contactos que este establece en los medios locales: allí estarán los miembros del Museo Social, el vicepresidente de la República y varios ministros de gobierno, el rector de la Universidad de Buenos Aires y el Presidente de la Universidad de la Plata, muchos universitarios, altos funcionarios y miembros de las colectividades migratorias. Los representantes de las tres principales colonias de extranjeros - italiana, española y francesa - profesarán incluso un pequeño discurso de despedida: el Dr. Laure lo hará en nombre de los franceses, el señor Teddeschi en nombre de los italianos, y López de Gomara de los españoles ${ }^{71}$.

${ }^{68}$ Ver «M.Mabilleau, sus conferencias. Su visita a El Hogar Obrero», en La Vanguardia (lunes 19-martes 20 de agosto, 1913): 1, col. 1.

${ }^{69}$ El Pueblo, 10/8/1912.

${ }^{70}$ En el mes de agosto de 1912 podemos encontrar casi periódicamente noticias sobre las actividades académicas y sociales de Mabilleau en Argentina. En un artículo sobre «La unión de mutualidades francesas», el periodista recuerda que el periódico había emprendido siete años atrás una campaña en pro de la federación de las sociedades de socorro mutuo francesas. Ver Le Courrier de la Plata (28/8/12): 3, col. 1-2. Sobre este periódico, ver Oteiza, 2000. Sobre la colectividad francesa, ver Otero, 2012.

${ }^{71}$ Ver Le Courrier de la Plata (9/8/1912): 3, col. 5-6; 4, col. 1-3; El Diario (7/9/12): 1, col. 5. Una biografía de López de Gomara en vida por José Citriniti en López de Gomara, 1914. Ver también Cutolo, 1975: 254-255. 
El prestigio de Léopold Mabilleau, no solo como especialista del tema de las mutualidades y hombre político francés, sino igualmente como intelectual bien insertado en redes académicas internacionales, juega sin lugar a dudas un importante papel en el renovado interés que van a manifestar los sectores reformistas por las sociedades de socorro mutuo y su decisión de movilizarlas en torno a su proyecto de creación de un mutualismo moderno ${ }^{72}$. Así, pocos meses después de regresar este a Francia, se reúne en Buenos Aires el Primer Congreso de la Confederación española en Argentina, convocado por iniciativa del inquieto y prolífico López de Gomara, quien había participado al banquete de despedida de Mabilleau ${ }^{73}$. Y allí Benjamín del Castillo, una figura del catolicismo social vinculado al Museo Social e implicado también en el tema del mutualismo, presenta un proyecto de «Federación de Sociedades Españolas de Socorros Mutuos» ${ }^{74}$. Pero a diferencia de las iniciativas previas de unión, este proyecto responde a normas internacionales de federaciones mutuales ${ }^{75}$. En él se exige la exoneración completa de los impuestos fiscales o municipales y la creación de subsidios públicos para las sociedades ${ }^{76}$. Ello constituye sin duda una novedad. La argumentación sobre la cual funda el reclamo se centra en la idea de «servicio» que estos prestan a la Comuna: «Me refiero, como lo habrán advertido mis honorables colegas, al servicio médico gratuito y al de los medicamentos, proporcionados por las sociedades a sus innumerables

${ }^{72}$ Mabilleau era ex-alumno de la Escuela Normal Superior, institución que formaba a las elites de la República. Su vida académica muestra que dispone de una amplia red de relaciones con las más prestigiosas instituciones. En 1902 hará un viaje a Estados Unidos y será invitado a impartir conferencias en las Universidades de Yale, Harvard, Columbia, Princeton, Chicago, New York, John Hopkins, Michigan, California, Pensilvania, Boston, Pittsburg, etc. En 1903 reitera su viaje a Estados Unidos y a Cuba. Ver Baltimore American (10/2/1903): 5; Trenteon Evening Times, (5/3/1903, New Jersey): 3; Springfield Sunday Republican (3/5/1903): 6; Diario de la Marina (La Habana, 23/4/1903): 4, col.1.

${ }^{73}$ Participan representantes de sociedades muy diversas, como entidades financieras españolas (Banco de Castilla, Banco de España, Banco Español del Río de la Plata, Banco de Galicia, Banco de Valencia y Aragón), sociedades culturales (Asociación patriótica Española, Ateneo Hispano-Americano), centros socio-culturales regionales (Centro Catalán, Orensano, Navarro, Gallego, Asturiano, etc.), sociedades de beneficencia y sociedades de socorros mutuos (las más numerosas, más de cien sociedades de socorros mutuos representadas). Ver Primer Congreso de la Confederación española en Argentina (PCCEA). Buenos Aires, 1913: 13-15.

${ }^{74}$ Benjamín E. del Castillo se presentaba como experto, habiendo ya publicado un trabajo sobre el tema. Ver Castillo, 1900: 250. Una referencia a la vinculación de del Castillo con el catolicismo social en Pike, 29/1 (1972): 1-16

${ }^{75}$ Precisa del Castillo que se trata de las resoluciones adoptadas por el Congreso de Lieja y el Congreso de Roma. Ver PCCEA.

${ }^{76}$ Ver el capítulo 1 del Estatuto de la Federación, en PCCEA: 96-10. 
asociados, constituyendo este servicio un sistema ya perfeccionado de hospitalización desde que todo afiliado a estas asociaciones de Socorros Mutuos no recurren a los Hospitales Municipales para su curación» ${ }^{77}$. Dos afirmaciones no totalmente exactas sustentan la argumentación: que la Asistencia Pública debía garantizar un seguro médico gratuito a todos los habitantes y que las SSM la remplazaban en el suministro de este servicio, lo que justificaba el reclamo de subvención ${ }^{78}$. En el centro de la argumentación se encuentra la noción, capital para Léon Duguit, de «servicio público» que podían cumplir las sociedades particulares ${ }^{79}$. Este no sólo desdibuja la frontera liberal entre individuo y Estado, sino que coloca la acción de los extranjeros en el campo de la república social. Cierto es que el proyecto, aceptado por la honorable asamblea, no pasó más allá de su recinto. Pero este episodio da cuenta de cómo se introducen entre las elites de la colectividad española una serie de nuevos argumentos, fundados en un nuevo vocabulario que vehicula nuevos conceptos, que la dirigencia del socorro mutuo - nacional y extranjero- comienza a compartir con los reformistas locales, y que los inserta en un vasto espacio de circulaciones transatlánticas de saberes sobre la sociedad. En torno a ellos se consolidan redes transnacionales de expertos que generan nuevos espacios internacionales de legitimación ${ }^{80}$. Del Castillo juega aquí claramente el papel de «conector» entre sociedades de socorro mutuo, sectores reformistas locales y redes transnacionales de intelectuales y expertos ${ }^{81}$. Y aunque no podemos dejar de considerar los escasos efectos concretos, tampoco podemos sortear el hecho de que este vocabulario compartido otorga a la dirigencia del socorro mutuo nuevas herramientas para negociar con las autoridades públicas, como lo demuestra la campaña que lanza el Museo Social simultáneamente a la tenida del Congreso sobre federación de sociedades españolas.

La movilización surge como respuesta a la decisión del procurador Julio Botet de rechazar la personería jurídica a ciertas sociedades de socorro mutuo. La importancia que toma este hecho banal es un primer testimonio de un

77 Ver PCCEA: 102.

${ }^{78}$ Las sociedades suministraban un servicio únicamente para aquellos que pagaban una cuota y los servicios de los hospitales de la Asistencia Pública eran únicamente gratuitos para aquellos inscriptos en el registro de pobres.

79 Ver Duguit,1901; 1911. Sobre su visita, ver en este mismo dossier el artículo de Zimmermann.

${ }^{80}$ Del Castillo justifica su posición diciendo que ella se inspira en las resoluciones adoptadas por el Congreso de Lieja y el Congreso de Roma respecto a la creación de federaciones Ver PCCEA, 107.

${ }^{81}$ Sobre regímenes circulatorios en el campo social ver Rodgers, 1998. Saunier, 71 (2008): 4-25; 57 (2004): 110-126. 
cambio de sensibilidad social. Pocas sociedades tenían personería jurídica, y ello se debía menos al rechazo de las autoridades que a la indiferencia que el medio asociativo manifestaba hacia la adquisición de este estatuto jurídico. Pero según el Museo, la prédica de Mabilleau había generado una nueva dinámica que impulsaba un cambio de actitud de las sociedades respecto a los poderes públicos ${ }^{82}$. Sea como fuere, Botet habría fundado su rechazo sobre el carácter «particular» que tienen las SSM, razón por la cual estas no podían atenerse a la condición de realizar el «bien común» según lo exigía el artículo 33 del Código Civil ${ }^{83}$. Así, casi simultáneamente a la celebración del congreso de los españoles, se libra en el campo político y jurídico nacional un similar combate en torno al reconocimiento del beneficio que las mutuales prestan a la colectividad. Frers utiliza un argumento similar a del Castillo: «las sociedades no solo son útiles para sus asociados, sino que haciéndolo lo hacen a la sociedad contribuyendo "eficazmente a la obra de la solidaridad social por la ayuda mutua" $\gg{ }^{84}$. Raymundo Wilmart, que participa a este debate, ahonda en el argumento de Del Castillo sobre las asociaciones «que disminuyen la carga del Estado» ${ }^{85}$. Más allá de las diferencias evidentes que podemos encontrar entre la visión de Frers, fundada sobre la obligación moral que se impone a los trabajadores y la posición de los socialistas reformistas como un Raymundo Wilmart que pone el acento en el beneficio para los trabajadores, la campaña da cuenta del interés que presenta el mutualismo para los sectores reformistas, así como de los vínculos que existen entre estos sectores y la dirigencia de las colectividades extranjeras implicadas en el socorro mutuo. El periódico francés Le Courrier de la Plata participa en este debate, utilizando los mismos

${ }^{82}$ Ver el Boletín del Museo Social Argentino (BMSA), 15-16 (1913).

${ }^{83}$ Los términos del ministro que retoma Frers en su carta son los siguientes: «limitando esas asociaciones, sus beneficios a sus propios asociados, que deben ser personas de determinados gremios, o nacionalidades, o tan solo nativos o descendientes de nativos de tal o cual provincia, generalmente de país extranjero, no son instituciones que realicen el bien común, como el código civil exige en su artículo 33». Ver BMSA, 15-16.

${ }^{84}$ «Que estas sociedades, si bien es cierto que directamente solo benefician a sus asociados, no puede de ahí deducirse que no tenga por principal objeto el bien común, puesto que muchas de ellas tienden a fomentar principios de moral social, de ahorro contra el pauperismo, de orden y trabajo contra el socialismo, de caridad para con los enfermos, que de otro modo acudirían a pedir amparo a los hospitales públicos, a lo que se agrega que, por el hecho de que hayan podido subsistir y prosperar por mucho tiempo, sin la personería jurídica no se sigue que deba negarse esa personería cuando ella se relama para que la corporación pueda tener la capacidad legal necesaria para adquirir y enajenar bienes raíces, sin recurrir al expediente siempre inseguro de tener los bienes sociales a nombre de los particulares». Ibidem: 116-117.

${ }^{85}$ Wilmart, 17 (Buenos Aires, 1913). Una biografía de R. Wilmart se encuentra en Tarcus, 2007. 
argumentos y vocabulario: las sociedades brindan un servicio a la comunidad, «realizando eficazmente una de las altas funciones del Estado, que es la de estimular "el desarrollo de la solidaridad social" ${ }^{86}$. La campaña fue un éxito. Las sociedades obtendrán la personería jurídica gracias a la intervención del nuevo ministro de Justicia, Carlos Ibarguren, quien aprovechará de la presencia de Léopold Mabilleau, nuevamente en Buenos Aires entre agosto y octubre de 1913, para presentar en septiembre un proyecto de ley orgánica de Sociedades de Socorro Mutuo ${ }^{87}$.

Si damos crédito a las memorias de Carlos Ibarguren, el francés lo habría asesorado para la formulación de esta primera iniciativa de legislación sobre mutualidades en Argentina. Según el mensaje que precede el proyecto de ley, el gobierno buscaba diseñar un sistema de asistencia y previsión que implicara a los interesados, evitando con ello «los sistemas providenciales, inspirados en la caridad, sea en doctrinas derivadas de la omnipotencia del estado» ${ }^{88}$. Se trataba de dar una respuesta alternativa a la que ofrecían las organizaciones obreras, proponiendo un modelo de seguro fundado en la «concordia social». Para ello, el texto reconoce dos tipos de socios, los participantes y los honorarios, permitiendo de este modo perpetuar el mecanismo de tutelaje de las elites sobre las sociedades obreras ${ }^{89}$. Pero el proyecto de ley prevé, bajo ciertas condiciones, la subvención pública de las SSM. Ello implica no solo que se reconozca el servicio que las sociedades cumplían sino también los derechos que tiene «la masa trabajadora» de estar provistas de un auxilio ${ }^{90}$. Adivinamos aquí en filigrana la doctrina solidarista. La prédica de Mabilleau también se deja ver en la implementación, como contrapartida de la subvención pública, de una inspección financiera del Ministerio de Justicia. Esto buscaba introducir un grado de tecnicidad en el cálculo de la cuota y la previsión del gasto, temas centrales para el movimiento mutualista internacional. El proyecto de ley murió en los archivos del congreso, según Ibarguren a causa de «la desgraciada cir-

${ }^{86}$ Ver Le Courrier de la Plata (8/8/1913): 3, col.1-2.

87 Ver BMSA, 23 (1914): 453-463.

${ }^{88}$ Ver Ibarguren, 1999: 263-264. Es de destacar que Ibarguren no se encuentra entre los primeros promotores del Museo Social y los primeros contactos con el ministro que esta institución deja registrados datan de 1913 cuando el Museo Social lleva a cabo la campaña en pro de la obtención de personería jurídica para las sociedades de socorros mutuos. Los fundamentos del Proyecto de Ley de 1913 en «Proyecto de ley orgánica sobre sociedades de socorro mutuo», Antecedentes legislativos sobre mutualidades en la República Argentina: 435-440.

${ }^{89} \mathrm{Si}$ esto efectivamente funcionaba así, el sugestivo trabajo de Gandolfo demuestra que ello no inhibía completamente ni la conciencia de clase ni la capacidad de acción de los obreros contra los protectores que podía ser también los explotadores.

${ }^{90}$ Ver BMSA, 23 (1914): 453-463. 
cunstancia» de la enfermedad y muerte del presidente Sáenz Peña, que apoyaba el proyecto ${ }^{91}$. Olvida mencionar en sus memorias la reticencia que su proyecto generaba entre ciertos sectores mutualistas, sin mencionar la clara hostilidad de los socialistas ${ }^{92}$. No más tarde de un año, el diputado socialista Ángel Giménez presentará otro proyecto de ley orgánica de mutualidades, con una suerte similar. En este último los socios honorarios pierden todo poder de gobierno. El texto introduce también mayores precisiones en lo que respecta al papel regulador del estado y a las exigencias de representatividad de los asociados y de tecnicidad en la gestión de los fondos ${ }^{93}$. A pesar de estas diferencias, ambos proyectos buscan responder a una necesidad/derecho de los trabajadores a través de formas de seguro solidarias, fundadas sobre la previsión de los trabajadores y el subsidio del Estado. Este consenso no caracteriza únicamente a los partidos políticos de gobierno. Como vimos, también lo comparten los sectores reformistas implicados en la experiencia del Museo Social. La prédica mutualista, y con ella todo un nuevo vocabulario sobre lo social, ingresa en las sociedades de socorro mutuo. En el caso de las francesas, la presencia del «apóstol de la mutualidad» y compatriota tuvo un impacto aún mayor. Durante su primer viaje se constituyó un "Comité Mabilleau» con el objeto de continuar con la prédica mutualista. Un año más tarde, durante la segunda estadía del experto en Buenos Aires, este es invitado a la ceremonia de creación de la Federación de Mutualidades Francesas, compuesta de 34 sociedades mutualistas francesas en Argentina, ceremonia en la que participa, junto a las autoridades consulares y las elites de la colectividad francesa, Carlos Ibarguren ${ }^{94}$.

Ese mismo año comienzan los preparativos para organizar en Argentina el Congreso Internacional de Mutualismo y Previsión Social, que había sido previsto, según Del Castillo, para 1915, pero que la guerra en Europa obligó a postergar un año, haciéndolo coincidir con los festejos del Centenario de la Independencia ${ }^{95}$. El congreso se organizó en tres secciones: Mutualidad,

\footnotetext{
91 Ver Ibarguren, 1999: 271-272.

92 Ver por ejemplo los comentarios de la Obrera «La futura ley, si no sufre modificaciones, engrosará las cajas de las instituciones que solo aspiran a levantar monumentales edificios sociales y mantener un enjambre de empleados». La Mutualidad, XI/9 (1913) en Belmartino, 2005: 30. La oposición de los anarco-sindicalistas ha sido destacada por Helene Finet en una tesis en curso sobre cultura contestataria en Argentina. Finet, 2010.

93 «Proyecto de ley orgánica de las mutualidades» en Antecedentes legislativos sobre mutualidades en la República Argentina, Buenos Aires, 1945: 459-469.

${ }^{94}$ Le Courrier de la Plata (21/09/13), (30/09/13).

${ }_{95}$ Congreso Internacional de Mutualidad y Previsión Social celebrado en Buenos Aires del 19 al 23 de julio de 1916 (CIMPS), Buenos Aires, Talleres Gráficos del Ministerio de Agricultura de la nación, 1917.
} 
Cooperativismo y Previsión Social. La comisión designada para coordinar la primera estaba constituida por los infatigables Carlos Ibarguren y Benjamín del Castillo, acompañados por Belisario J. Montero, un diplomático y periodista que durante su estadía en Bélgica, en calidad de Cónsul General, había estudiado los sistemas de seguro en Europa ${ }^{96}$. Junto a ellos figuran algunos notables y políticos provinciales como los hermanos Carbó, y miembros prominentes de las colectividades extranjeras implicadas en la vida asociativa, como Basilio Cittadini ${ }^{97}$. Entonces vuelven a abordarse tres principales puntos que figuraban tanto en el proyecto de federación de los españoles como en los proyectos de ley sobre mutualidades, y que dan cuenta de la instauración de un espacio de encuentro e intercambio entre la dirigencia de las sociedades de socorro mutuo, los sectores reformistas y los políticos más o menos implicados en los movimientos de reforma. Constatamos allí un cierto acuerdo negociado en torno a los reclamos de subsidio público ${ }^{98}$, la exigencia de mayor rigor financiero de las sociedades y la necesidad de promover federaciones nacionales. También da cuenta de lo que aún no genera consenso y que hace al principio de la obligatoriedad de la cotización para los trabajadores, como se desprende de la discusión del proyecto presentado por el Dr. Enrique Feinmann sobre «Previsión social de la tuberculosis, la higiene social y la defensa social de la salud, y del trabajo como organización mutualista ${ }^{99}$. Pero vemos ya una importante inflexión en la objeción liberal a un derecho al socorro que el Estado debe garantizar ${ }^{100}$. Hilarión Larguía, presidente de la Caja Nacional de Jubilaciones y Pensiones, justifica la subvención pública a través de la noción de «deuda social», citando explícitamente a Léon Bourgeois y declarando: «Ya es tiempo de abandonar el laissez passer, laissez faire, laissez mourir de los

96 Montero, 1905.

97 Sobre lidearzgo étnico en Argentina ver Bernasconi y Frid, 2006. El papel de Cittadini en la colectividad italiana en Cibotti, 1992; González Bernaldo de Quirós, 2009: 115-125.

${ }_{98}$ Así la asamblea aceptó el proyecto presentado por José R. Salvá sobre «Principio fundamental sobre el mutualismo argentino» rechazando el artículo que declaraba «la mutualidad no necesita la ayuda material del gobierno, pero debe ser protegida por leyes que la fomenten». Ver «Crónica» en CIMPS: 32.

99 Ver «Crónica» en CIMPS: 29-30.

${ }^{100}$ La objeción liberal al principio de derecho al socorro era muy fuerte. Alexis de Tocqueville en su Mémoire sur le paupérisme de 1835 va a ofrecer una serie de argumentos que utilizarán los liberales argentinos durante la segunda mitad del siglo XIX respecto a los inconvenientes de desarrollar un derecho al socorro a partir del cual se instalaría lo que Tocqueville califica de «caridad pública» y que tendría como consecuencia nefasta desarrollar una sociedad de asistidos. En este marco el socorro mutuo como previsión libre constituía una respuesta adecuada, pero no implicaba ni la subvención ni menos aún la obligatoriedad. Sobre este tema ver el clásico trabajo de Ewald, 1986. 
economistas que entregaban a la caridad pública la solución planteada por la indigencia, para remplazarla por la solidaridad, que es índice de alto grado de cultura y que muestra que el necesitado puede recibir la ayuda sin sonrojarse y sin depender de la voluntad del filántropo y como un derecho resultante de una deuda social y de una conveniencia de la mayoría» ${ }^{101}$.

El congreso internacional motivó aún más a los diferentes actores locales para transformar al socorro mutuo en sistema de seguro social. Ello requería previamente la sanción de una ley de mutualidades. Para ello el Museo Social decide organizar un congreso con el objeto de discutir con todos los actores implicados un texto de ley sobre las mutualidades que sería luego propuesto al voto del Congreso de la Nación ${ }^{102}$. Se convoca así el primer Congreso de Mutualidad en Argentina, celebrado en Buenos Aires en marzo de $1918^{103}$. Aquí volvemos a encontrar a Carlos Ibarguren, ahora acompañado del socialista y médico higienista Augusto Bunge ${ }^{104}$, junto con Benjamín Larroque, presidente de la Sociedad Española de Socorros Mutuos, y Enrique Ruiz Guiñazú, jurista y profesor de la Universidad de Buenos Aires que dio una particular acogida a las propuestas del profesor Duguit ${ }^{105}$. La composición de la comisión refleja las diversas corrientes ideológicas, grupos profesionales y sectores sociales y políticos que alimentan el debate sobre mutualidad y seguro social. Ello muestra

101 Ver «La Previsión Social. Informe presentado a la Comisión de legislación de la H. Cámara de Diputados de la Nación por el Dr. Hilarión Larguía, Presidente de la Caja de Jubilaciones y Pensiones» en CIMPS: 68. Ver también Zimmermann, 1995: 77-78.

102 Ver BMSA, 69-70 (1917): 558-560.

103 Ver Congreso de la mutualidad organizado por el Museo Social Argentino y celebrado en la ciudad de Buenos Aires durante los dias 24-25 y 26 de marzo de 1918. Buenos Aires, 1918.

${ }^{104}$ Augusto Bunge, (25/4/1877 a 1/8/1943) es médico, hermano de Carlos Octavio, Alejandro Bunge y Delfina Bunge, y cuñado de Manuel Gálvez. Ingresa en Centro Socialista Universitario en 1896 y al PS en 1897. Diputado socialista en 1916, renueva su banca hasta 1936. En octubre 1920 fundó el periódico LA HORA que defendió posiciones de la III Internacional al interior del PS. Entre los proyectos presentados en legislatura se cuenta los de seguro nacional, de reforma de la ley de accidentes de trabajo, de Caja Nacional de Jubilaciones y de restablecimiento de relaciones diplomáticas y comerciales con URSS. Ver Antecedentes y legislación sobre Mutualismo en la República Argentina (ALMRA), Buenos Aires, 1945: 441-457. Será el autor del «Proyecto de ley de Mutualidades y Seguros Populares» presentado el 22 de septiembre de 1919 a la H. Cámara de Diputados. Nota biográfica de A. Bunge en Tarcus, 2007.

${ }^{105}$ La ausencia de Del Castillo puede explicarse por el diferendo que lo oponía al Museo Social y que había hecho público durante su conferencia de apertura del Congreso Internacional de 1916. Es probable que esta delicadeza no haya sido bien apreciada por los directivos del Museo Social. Sobre la intervención de Ruiz Guiñazú en el debate que generarán las conferencias de Duguit en Buenos Aires ver el artículo de E. Zimmermann, «Un espíritu nuevo»: la cuestión social y el Derecho en la Argentina, 1890-1930» en este mismo número. 
que se han tejido ya en Argentina interacciones duraderas entre actores mutualistas y la «nebulosa solidarista». Podemos ciertamente advertir posiciones divergentes entre un conservador como Ibarguren, que insiste en representar su proyecto de ley sobre mutualidades de 1913, que busca la «armonía social», un socialista como Augusto Bunge, que defiende junto con otros responsables de sociedades de socorro mutuo el principio de obligatoriedad «para todo aquel cuya economía dependa de un salario», un mutualista como Larroque, que reclama la subvención pero la funda en el sentimiento de filantropía, y un sindicalista como A. Comolli, que responde en términos de protección como derecho. Constatamos sin embargo avances respecto al debate en el congreso de 1916. El proyecto que presenta Bonelli, delegado de la sociedad «La Argentina» sobre obligatoriedad de la adhesión a los socorros mutuos obtiene entonces un amplio apoyo ${ }^{106}$. Junto a la obligatoriedad aparece de manera más nítida la cuestión de la tecnicidad de las sociedades que requiere la intervención de especialistas en matemáticas actuariales. Según Argentino Acerboni, 25\% de las sociedades de socorro mutuo con las que se quiere construir un sistema de protección social tiene esa defíciencia técnica en su base ${ }^{107}$. Desde su punto de vista, sin una intervención de los actuarios, el edificio diseñado para proteger a los trabajadores está destinado a desmoronarse. Poco sabemos sobre esta corporación y el papel que jugará en el pulso que se dará luego entre SSM y sindicatos en torno al seguro social de los trabajadores, pero ya hacia los años 20 la ecuación del seguro integra a los actuarios.

El proyecto de ley que propondrá Augusto Bunge al Congreso de la Nación en 1919 se inspira de las proposiciones y debates de este congreso. En este, Bunge no reduce el seguro popular a las SSM, pero integra todas las funciones que cumplían las SSM dentro de la definición del seguro popular como «toda forma de previsión de los riesgos de enfermedad, maternidad, desocupación, invalidez, vejez, defunción y orfandad practicadas mediante cotizaciones periódicas de los aspirantes...» ${ }^{108}$. Cierto es que el proyecto conoció

106 Dos posiciones se enfrentan aquí sin embargo: la de Ibarguren y Bonardi, que parece entonces minoritaria, y la de Bunge que va a inspirar el proyecto de ley sobre mutualidades de 1919. En respuesta a la objeción liberal al principio de obligatoriedad, Argentino Acerbini responde: «Si bien tenemos el derecho de no ser mutualistas si no lo deseamos, como tenemos muchos derechos de esa clase, no tenemos en cambio el derecho de cargar a la sociedad con la culpa de nuestras imprevisiones». Ibidem: 242.

${ }^{107}$ Intervención de Argentino Acerboni que participa en representación de la Dirección General de Estadística y Departamento Provincial de Trabajo de La Plata. Acerboni es el primer especialista local de las matemáticas actuariales. Había defendido en 1916 una tesis sobre «Fundamentos matemáticos de los seguros sociales» en la Facultad de Economía. CIMPS: 119-120.

108 Proyecto de Ley de 1919, art.1: 441. 
la misma suerte que los precedentes, y habrá que esperar la iniciativa tomada por Juan Domingo Perón de reunir, en 1944, desde la secretaría de Trabajo y Previsión, un nuevo congreso de mutualidades con el fin de discutir una ley de mutualidad que llevaría a un «Mutualismo integral, controlado por el Estado, subsidiario del seguro social» ${ }^{109}$. Pero el principio de subsidio al sistema de previsión solidaria, que parece haber funcionado de manera informal, fue reglamentado ${ }^{110}$. Finalmente, como sabemos, Perón optará por colocar a los sindicatos en el centro del sistema de cajas de previsión. Lo que llevará a un debilitamiento y desaparición gradual de las sociedades de ayuda mutua.

\section{CONSIDERACIONES FINALES}

Son sin duda los sucesivos fracasos legislativos los que sellaron el destino de este «momento mutualista», condenándolo al olvido. Por razones de espacio y de economía de la argumentación no entré aquí en el análisis de las razones que puedan explicar estos fracasos. Es evidente que ni los grupos representados en el Congreso nacional parecen considerar el tema de excesiva necesidad o interés, ni los representantes de las mutualidades tienen suficiente poder para imponerlo, o gran interés de hacerlo. Debemos también considerar, como lo sugiere S. Belmartino, que la presentación de un proyecto a la Asamblea puede perseguir objetivos múltiples que no requieren necesariamente que este sea aprobado $^{111}$. Esto no es por otro lado una especificidad local, y encontramos en países que son citados como modelos, como la propia Francia, similares diacronías entre los primeros intentos de reforma legislativa y su efectiva aplicación. La especificidad local proviene en cambio de la importancia de las sociedades de socorro mutuo de los inmigrantes en el conjunto del movimiento mutualista. Pero esto tampoco es una novedad, y el propio Borea ya lo señala en el censo de 1914. Sin embargo, los actores de entonces y los estudios posteriores tienden a considerar este hecho como un freno a la democracia

\footnotetext{
109 Conferencia del Sr. Subdirector General de Previsión social, de la secretaría de trabajo y previsión, Roberto Palmieri, en ALMRA.

${ }^{110}$ Ley Nacional n. ${ }^{\circ} 12.209$ sobre exención de pago de todo impuesto nacional a las asociaciones mutualistas, presentado por diputado nacional Fernando de Andreis, sancionada por el H.C. de la Nación el 24 de septiembre de 1935 y promulgada por el PE de la Nación el 1 de octubre de 1935 en ALMRA: 545-546. Las actas de la Municipalidad de Buenos Aires ofrecen varios ejemplos de concesión de reducción de impuestos a las sociedades de socorro mutuo y otras asociaciones benéficas.

111 Belmartino sugiere que la elaboración de proyectos de ley puede constituir «un elemento más del obligado cursus honorum de estos notables». Belmartino, 2005: 55.
} 
social, así como el desinterés de los extranjeros por la naturalización lo habría sido para la democracia política ${ }^{112}$. En este trabajo hemos demostrado que el carácter «extranjero» del socorro mutuo no fue un elemento que inhabilitó a los mutualistas para reclamar protección al Estado de acogida en nombre del servicio que ofrecían a la comunidad, como tampoco disuadió a los políticos para diseñar proyectos de seguro nacional fundados sobre el socorro mutuo extranjero. Las categorías nacionales que los estudios transnacionales denuncian como inadaptadas para entender el mundo globalizado no son tampoco pertinentes para comprender el proceso de construcción del Estado social, aunque este coincida con la consolidación política de los Estados nacionales. El estudio de este momento mutualista tiene así como primer interés el de poner en evidencia la dimensión transnacional de la construcción de un campo de lo social, campo que será sin embargo identificado con las fronteras políticas y jurídicas del Estado nacional ${ }^{113}$.

El caso aquí analizado ofrece también un ejemplo de articulación entre el mundo social, los nuevos conocimientos sobre lo social y la formulación de políticas de acción social. Abordar esta articulación supone que previamente aceptemos la polisemia de la noción de social/sociedad como un elemento constitutivo de la interacción. Un mundo puede separar a los adherentes de la pequeña sociedad de socorros mutuos catalana, que buscan a través de prácticas ancestrales soluciones pragmáticas a nuevos problemas, de los universitarios que desde la Universidad de Buenos Aires y de la más joven y audaz Universidad de la Plata buscan hacer de estas casas de estudios centros de producción científica de nuevos conocimientos, como de los políticos que buscan restablecer la paz social interviniendo en un conflicto entre capital y trabajo que los obliga a revisar las representaciones jurídicas de lo social como espacio de las relaciones civiles. El momento mutualista no allana estas diferencias, pero permite analizar cómo se articulan. La formulación de proyectos, aunque no se traduzca en leyes, genera nuevos espacios de debate entre elites reformistas y diferentes sectores del mutualismo, entre ellos una importante proporción de extranjeros que denota la existencia de un lenguaje común a partir del cual se comunican estos diferentes sectores. Vimos que este encuentro se funda en numerosos desacuerdos respecto a lo que se entendía por «derecho a la protección». Así, las visitas de intelectuales y especialistas extranjeros, la organización de congresos, el debate en la prensa periódica y en revistas especializadas, facilitan la difusión y

112 Un desarrollo de este punto en González Bernaldo de Quirós, 2009: 115-125.

${ }^{113}$ La bibliografía sobre este tema ya muy extensa pues el debate implica a varias disciplinas de las ciencias sociales. Ver Amelina, Nergiz, Faist y Glick Schiller, 2012. 
reapropiación de un vocabulario común que comparten los dirigentes mutualistas (nacionales y extranjeros) con los sectores reformistas, tanto liberales como conservadores y socialistas. Esto no allana las diferencias, pero ofrece un nuevo lenguaje para formularlas y testimonia los procesos sociales de construcción de representaciones comunes del campo social sobre el cual se busca intervenir.

\section{BIBLIOGRAFÍA Y FUENTES}

Alonso, Guillermo, Politica y seguridad social en la Argentina en los años 90. Buenos Aires, Niño y Dávila Ed. Clacso, 2000.

Amelina, Anna, Nergiz, Devrimsel D., Faist, Thomas y Glick Schiller, Nina, Beyond Methodological Nationalism, Research Methodologies for Cross-Border Studies, London, Routledge, 2012.

Andrews, George Reid, The Afro-Argentines of Buenos Aires 1800-1900, The University of Wisconsin Press, 1980.

Antecedentes y legislación sobre mutualismo en la República Argentina, Buenos Aires, Imp. de la Cámara de Diputados de la Nación, 1945.

Armus, Diego, La ciudad impura: salud, tuberculosis y cultura en Buenos Aires 1870-1950. Buenos Aires, Edhasa, 2007.

Armus, Diego, López Denis, Adrian, «Desease, Medicine, and Health», Moya, José, The Oxford Hadbook of Latin American History, Oxford University Press, 2011: 424-453.

Audier, Serge, La pensé solidariste. Aux sources du modèle social républicain. París, PUF, 2010.

Baily, Samuel, «Las sociedades de ayuda mutua y el desarrollo de una comunidad italiana en Buenos Aires, 1858-1918», Desarrollo Económico, 21/84 (1982): 485-514.

Belmartino, Susana, Bloch, Carlos, Persalli, Ana y Quiroga, Hugo, Las Instituciones de Salud en la Argentina. Desarrollo y crisis. Buenos Aires, Secretaria de Ciencia y Técnica. Área de Estudios e investigaciones en Ciencias Sociales para la Salud, 1987.

Belmartino, Susana, La atención médica argentina en el siglo XX: instituciones y procesos, Buenos Aires, Siglo XXI Editores, 2005.

Bernasconi, Alicia y Frid, Carina, De Europa a las Américas. Dirigentes y liderazgos (1880-1960), Buenos Aires, Ed. Biblos, 2006.

Boletín del Museo Social Argentino, Buenos Aires, Museo Social Argentino, números 1-102 (1912-1930). 
Boltanski, Luc, «Les usages sociaux du corps», Annales, Economie, Sociétés, Civilisations, 26/1 (janvier-février 1971): 205-233.

Borea, D., «La mutualidad y el cooperativismo en la República Argentina», Comisión Nacional del Censo, Tercer Censo Nacional, Buenos Aires, 1916-19.

Bourdé, Guy, Urbanisation et immigration en Amérique latine. Buenos Aires XIXe et XXe siècles. París, Aubier, 1974.

Bourgeois, Léon, La Solidarité, París, A. Colin, 1896.

Carnavaca, Jimena y Plotkin, Mariano, «Crisis, ciencias sociales y elites estatales en Argentina, 1910-1935», Desarrollo Económico. Revista de Ciencias Sociales, 47/187 (octubre-diciembre, 2007): 401-428.

Castel, Robert, La metamorfosis de la cuestión social. Una crónica del salariado. Buenos Aires, Paidós, 1998 (Fayard, 1995).

Castillo, Benjamín E. del, Mutualidad, Cooperativismo y previsión, Valencia, F. Sempere y Compañía Editorial, 1900.

Castro, Beatriz, «Las sociedades de ayuda mutua en Colombia», Anuario Colombiano de Historia Social y de la Cultura, 29 (2002): 195-221.

Censo General de la Población, Edificación, Comercio e Industria de la Ciudad de Buenos Aires, capital federal de la República Argentina, levantado en los días 11 y 18 de septiembre de 1904. Bs. As., Cía. de Billetes de Banco, 1906.

Cibotti, Ema, «Sufragio, prensa y opinión pública: las elecciones municipales de 1883 en Buenos Aires», Antonio Annino (coord.), Historia de las elecciones en Iberoamérica, siglo XIX, Buenos Aires, FCE, 1995: 143-175.

Congreso Internacional de Mutualidad y Previsión Social celebrado en Buenos Aires del 19 al 23 de julio de 1916. Buenos Aires, Talleres Gráficos del Ministerio de Agricultura de la nación, 1917

Congreso de la mutualidad organizado por el Museo Social Argentino y celebrado en la ciudad de Buenos Aires durante los días 24-25 y 26 de marzo de 1918. Buenos Aires, Oceana, 1918.

Courrier de la Plata (Le), Buenos Aires, 1911-1913.

Cutolo, Vicente Osvaldo, Nuevo Diccionario Biográfico Argentino (1750-1930), 4, $L-M$, Buenos Aires, Ed. Elche, 1975.

Devoto, Fernando, «Participación y conflictos en las sociedades italianas de socorros mutuos en Buenos Aires y Santa Fe», Estudios sobre la inmigración italiana en la Argentina en la segunda mitad del siglo XIX, Nápoles, Edizioni Scientifiche Italiane-Universita de Sassari, 1991: 144-166.

Devoto, Fernando, Movimientos migratorios, historiografía y problemas, Buenos Aires, CEAL, 1992. 
Devoto, Fernando, «La experiencia mutualista en la Argentina: un balance», Fernando Devoto y Eduardo Mínguez (eds.), Asociacionismo, trabajo e identidad étnica. Los italianos en América latina en una perspectiva comparada, Buenos Aires, CEMLA-CSER-IEHS, 1992: 169-188.

Devoto, Fernando, Le migrazioni italiane in Argentina. Un saggio interpretativo. Istituto Italiano per gli Studi Filosofici, Napoli, 1994.

Devoto, Fernando y Otero, Hernan, «Veinte años después. Una lectura sobre el crisol de razas, el pluralismo cultural y la historia nacional en la historiografía argentina», Estudios Migratorios Latinoamericanos, 17/50 (2003): 181-228.

Devoto, Fernando, Historia de los italianos en la Argentina, Buenos Aires, Ed. Biblos, 2006 (2 ed. 2008: 165-232).

Donzelot, Jacques, L'invention du social. Essai sur le déclin des passions politiques, París, Fayard, 1984.

Dreyfus, Michel, Gibaud, Bernard y Gueslin, André (dir.) Démocratie, solidarité et Mutualité. Autour de la loi de 1898, París, Economica, 1999.

Duguit, Léon, L'Etat, le droit objectif et la loi positive, París, Ancienne Librairie Thorin et fils, 1901.

Duguit, Léon, Le droit social, le droit individuel et la transformation de l'Etat. Conférences faites à l'école des hautes études sociales. París, F. Alcan Éd., 1911.

Ewald, François, Histoire de l'Etat providence. Les origines de la solidarité, París, Grasset, 1986.

Falcón, Ricardo, L'immigration, les travailleurs et le mouvement ouvrier en Argentine: 1870-1912, Tesis de tercer ciclo, París, EHESS, 1985.

Fassin, Didier, L'espace politique de la santé, París, Puf, 1996.

Fernandez, Alejandro, «El mutualismo español en Buenos Aires, 1890-1920. Un estudio de caso», Cuadernos de Historia Regional, III/8 (1987): 36-71.

Fernández, Alejandro, «Mutualismo y asociacionismo», AA.VV, Historia general de la emigración española a Iberoamérica, 1, Madrid, CEDEAL, 1992: 331-357.

Finet, Hélène, Pratiques culturelles anarchistes et sociabilités ouvrières en Argentine, 1900-1925. Tesis de doctorado en Historia de la Universidad Paris Diderot, París, 2010.

Forment, Carlos, Democracy in Latin America, 1760-1900, Chicago, University of Chicago Press, 2003.

Fouillée, Alfred, La propriété sociale et la démocratie, París, Hachette, 1884.

Gandolfo, R., «Las sociedades italianas de socorros mutuos de Buenos Aires: cuestiones de clase y etnia dentro de una comunidad de inmigrantes (1880-1920)», Fernando Devoto y Eduardo Mínguez (dir.), Asociacionismo, trabajo e identidad 
étnica. Los italianos en América latina en una perspectiva comparada, Buenos Aires, CEMLA, CSE, IEHS, 1992: 311-332.

García-Bryce, Iñigo, «Politics by Peaceful Means: Artisan Mutual Aid Societies in Mid-Nineteenth-Century Lima, 1860-1879», The Americas, 59/3 (enero, 2003): 325-345.

Germani, Gino, Estructura social de la Argentina. Análisis estadístico, Buenos Aires, Solar, 1987.

Godio, Julio, El Movimiento obrero y la cuestión social en Argentina: inmigrantes, asalariados y lucha de clases, 1880-1910, Buenos Aires, Ed. Erasmo, 1972.

González Bernaldo de Quirós. Civilité et politique, Aux origines de la Nation Argentine. Paris, Ed. de la Sorbonne, 1999; Buenos Aires, FCE, 2001.

González Bernaldo de Quirós, Pilar, «Mobilidade e protección: unha aproximación comparativa do desenvolvemento de formas de axuda mútua en Francia e Arxentina», Estudios Migratorios, Nueva época, 1, Santiago de Compostela, Arquivo de Emigración Galega, 2008: 81-117.

González Bernaldo de Quirós, Pilar, «Etrangers à la nation, citoyens dans la cité: l'expérience politique des étrangers dans la ville de Buenos Aires pendant la deuxième moitié du XIXe siècle», Pilar González Bernaldo de Quirós, Manuela Martini y Marie Louise Pelus-Kaplan, Etrangers et sociétés, PUR, 2008: 115-125.

González Leandri, Ricardo, González Bernaldo de Quirós, Pilar y Suriano, Juan, La temprana cuestión social. La ciudad de Buenos Aires durante la segunda mitad del siglo XIX, Madrid, CSIC, 2010.

Gueslin, André, L’invention de l'économie sociale, París, Economica, 1987.

Hale, Charles A., «Ideas políticas y sociales, 1870-1930», Leslie Bethell (ed.), Historia de América latina. Vol. 8: América latina: cultura y sociedad, 1830-1930, Cambridge University Press, Editorial Crítica, 1991: 1-64.

Horne, Janet, Le Musée Social. Aux origines de l'Etat Providencie, París, Belin, 2004: 384 .

Linden, Marcel Van Der, Dreyfus, Michel, Gibaud, Bernard y Lucassen, Jan, Social Security Mutualism: The Comparative History of Mutual Benefit Societies, Peter Lang Pub Inc., 1996.

Ibarguren, Carlos, La historia que he vivido, (1955), Buenos Aires, Ed. Sudamericana, 1999.

Lo Vuolo, Rubén y Barbeito, Alberto, La nueva oscuridad de la política social. Del Estado populista al neoconservador. Buenos Aires, Miño y Dávila Ed., Ciepp, 1994.

Lopez de Gomara, Justo S., De Ambas orillas del mar. Cuentos originales de ambiente hispanoargentino, Buenos Aires, 1914. 
Lvovich, Daniel y Suriano, Juan, Las politicas sociales en perspectiva histórica. Argentina, 1870-1952, Buenos Aires, Prometeo libros, UNGSAM, 2006.

Marotta, Sebastián, El movimiento sindical argentino, Buenos Aires, Ed. Lacio, 196061.

Mesa-Lago, Carmelo, Social Security in Latin America. Pressure Groups, Stratification and Inequality. Pittsburgh, University of Pittsburgh Press, 1978.

Montero, Belisario, Estudios Sociales, Bruselas, Weissenbruch Impresoras del Rey, 1905.

Moya, Cousins and Strangers. Spanish Immigrants in Buenos Aires, 1850-1930, Berkeley/Los Ángeles/, University of California Press, 1998.

Moya, José, «Immigrants and Associations: A Global and Historical Perspective», Journal of Ethnic and Migration Studies, 31/5 (September, 2005): 833-864.

Munck, Ronaldo, «Mutual Benefit Societies in Argentina: Workers, Nationality, Social Security and TradeUnionism», Journal of Latin American Studies, 30/3 (October, 1998): 573-590.

Niklison, José Elias, «Acción social Católica Obrera», Boletín del Departamento Nacional del Trabajo, 46, (Buenos Aires, marzo de 1920): 199-200.

Oddone, Juan, Gremialismo proletario argentino, Buenos Aires, La Vanguardia, 1949.

Oteiza, Vivianne, «Le Courrier de La Plata. Diario de la colectividad francesa rioplatense». Tesis de Maestría, UNSAM, 2000.

Otero, Hernán, Historia de los franceses en la Argentina. Buenos Aires, Ed. Biblos, 2012.

Palacios, Alfredo L., La Miseria (en la República Argentina), Buenos Aires (1900), Colegio Público de Abogados, 2004.

Passanante, María Inés, Pobreza y acción social en la historia argentina: de la beneficencia a la seguridad social, Buenos Aires, Editorial Humanitas, 1987.

Pelosi, Hebe Carmen, El Museo Social Argentino y la Universidad del Museo Social Argentino. Historia y Proyección (1911-1978), Buenos Aires, Universidad del Museo Social Argentino, 2000.

Pike, Frederic, «Spanish Origins of the Social-Political Ideology of the Catholic Church in Nineteenth-Century Spanish America», The Americas, 29/1 (July, 1972): 1-16.

Plotkin, Mariano Ben y Caravaca, Jimena, «A economía entre crises. Economia política e finanças na Universidade de Buenos Aires (1870-1900)», Tempo, Social. Revista de sociología da USP, 21/2, (noviembre, 2009): 87-108.

Plotkin, Mariano Ben y Zimmermann, Eduardo (comp.), Los saberes del Estado. Buenos Aires, Edhasa, 2012. 
Prensa (La), Buenos Aires, 1911-1913.

Pueblo (El), Buenos Aires, 1911-1913.

Recalde, Héctor, «La primera cruzada contra la tuberculosis, Buenos Aires, 1935», José Panetieri (comp.), Argentina: trabajadores entre dos guerras. Buenos Aires, Eudeba, 2000: 55-86.

Rodgers, Daniel T., Atlantic Crossings. Social Politics in a Progressive age, Cambridge, Masssachusetts, Harvard University Press, 1998.

Rosanvallon, Pierre, La crise de l'Etat Providence, París, Seuil, 1981.

Rosanvallon, Pierre, La nouvelle question sociale, Repenser l'Etat-Providence, París, Seuil, 1995.

Sabato, Hilda, «El pluralismo cultural en la Argentina: un balance crítico», Comité Internacional de Ciencias Históricas, Historiografía Argentina. Una evaluación crítica de la producción histórica argentina, Buenos Aires, 1990: 350-366.

Sabato, Hilda y Cibotti, Ema, «Hacer política en Buenos Aires: los italianos en la escena pública porteña, 1860-1880», Boletín del Instituto de Historia Argentina y Américana Dr. E Ravignani, Tercera Serie (2ºmestre, 1990): 7-46.

Saunier, Pierre-Yves, «Les régimes circulatoires du domaine social 1800-1940: projets et ingénierie de la convergence et de la différence», Genèse 71 (juin, 2008): 4-25.

Saunier, Pierre-Yves, «Circulation, connexion et espaces transnationaux» Genèse 57 (déc. 2004): 110-126.

Segundo Censo de la República Argentina, mayo 10 de 1895. 3 tomos. Buenos Aires, Taller Tipográfico de la Penitenciaria Nacional, 1898.

Societat Catalana de Socors Mutuos, Memoria que la comissió directiva del MontePio de Montserrat 1880-1930, Buenos Aires, 1905.

Sordo Cerdeño, Reynaldo «Sociedades de Socorros mutuos en México 1867-1880», Historia Mexicana, 33/1 (129) (julio-septiembre, 1983): 72-96.

Suriano, Juan, (comp.), La cuestión social en Argentina 1843-1943, Buenos Aires, La Colmena, 2000.

Tarcus, H. (dir.), Diccionario biográfico de la izquierda argentina, Buenos Aires, Emecé, 2007.

Tercer Censo Nacional, levantado el 1 de junio de 1914, 10 tomos, Buenos Aires, Talleres Gráficos de L. J. Rowsso, 1916-1919.

Thompson, Andrés, «El Tercer Sector en la Historia Argentina», Buenos Aires, CEDES, 1994.

Tocqueville, Alexis de, Mémoire sur le paupérisme (1835), París, Allia, 1999. 
Zimmermann, Eduardo, Los liberales reformistas: la cuestión social en la Argentina, 1890-1916, Buenos Aires, Editorial Sudamericana, Universidad de San Andrés, 1995.

Zimmermann, Eduardo, «Libéralisme et sciences sociales en Argentine à la fin du dix-neuvième siècle: des Lumières à la nouvelle "connaissance sociale"», Amérique latine Histoire et Mémoire. Les Cahiers ALHIM, 11, 2005.

Fecha de recepción: 15/11/12

Fecha de aceptación: 28/2/13

\section{The «mutualist moment» in the formulation of a social welfare system in Argentina: mutual aide and subsidized prevention at the beginning of the 20th century.}

In this article I study the foreigners' mutual aid practices and their role in the diffusion of solidarist thinking that would provide a set of conceptual tools and techniques that would be essential in the demands for protection coming from workers and in formulating the first projects for putting in place a national insurance system. This research allow me to point out the transnational dimension of the construction of the field of social protection. The first part of the article is an empirical demonstration of this reality using qualitative and quantitative sources from public and private archives. In the second one I pay attention to the concrete mechanisms in the circulation of knowledge. In particular, the article analyses the different mutualist congresses organized in Buenos Aires during the first two decades of the twentieth century and the impact of the visit of Léopold Mabilleau, a French intellectual, expert in mutual insurance systems and "apostle» of solidarism.

KeY-words: Mutual aid; Solidarism; Circulation of knowledge; Social state; Argentina; $X I X-X X$ century. 